\title{
Cell Fate Decisions in the Wake of Histone H3 Deposition
}

\author{
Reuben Franklin, Jernej Murn* and Sihem Cheloufi* \\ Department of Biochemistry, Stem Cell Center, University of California, Riverside, Riverside, CA, United States
}

OPEN ACCESS

Edited by:

Justin Brumbaugh,

University of Colorado Boulder,

United States

Reviewed by:

Andrew P. Hutchins,

Southern University of Science

and Technology, China

Genevieve Almouzni,

UMR 3664 Dynamique du noyau,

France

*Correspondence:

Jernej Murn

jernej.murn@ucr.edu

Sihem Cheloufi

sihem.cheloufi@ucr.edu

Specialty section:

This article was submitted to

Stem Cell Research,

a section of the journal

Frontiers in Cell and Developmental

Biology

Received: 17 January 2021

Accepted: 18 March 2021

Published: 20 April 2021

Citation:

Franklin R, Murn J and Cheloufi S (2021) Cell Fate Decisions

in the Wake of Histone H3 Deposition.

Front. Cell Dev. Biol. 9:654915.

doi: 10.3389/fcell.2021.654915
An expanding repertoire of histone variants and specialized histone chaperone partners showcases the versatility of nucleosome assembly during different cellular processes. Recent research has suggested an integral role of nucleosome assembly pathways in both maintaining cell identity and influencing cell fate decisions during development and normal homeostasis. Mutations and altered expression profiles of histones and corresponding histone chaperone partners are associated with developmental defects and cancer. Here, we discuss the spatiotemporal deposition mechanisms of the Histone $\mathrm{H} 3$ variants and their influence on mammalian cell fate during development. We focus on $\mathrm{H} 3$ given its profound effect on nucleosome stability and its recently characterized deposition pathways. We propose that differences in deposition of $\mathrm{H} 3$ variants are largely dependent on the phase of the cell cycle and cellular potency but are also affected by cellular stress and changes in cell fate. We also discuss the utility of modern technologies in dissecting the spatiotemporal control of $\mathrm{H} 3$ variant deposition, and how this could shed light on the mechanisms of cell identity maintenance and lineage commitment. The current knowledge and future studies will help us better understand how organisms employ nucleosome dynamics in health, disease, and aging. Ultimately, these pathways can be manipulated to induce cell fate change in a therapeutic setting depending on the cellular context.

Keywords: chromatin, histone H3, nucleosome diversity, histone chaperone, cell cycle, reprogramming, development, cellular plasticity

\section{INTRODUCTION}

\section{The Nucleosome and the Histone H3 Family}

Cell fate decisions are central to development, normal homeostasis, and responding to infections, injury, and aging. During these processes, stem cells sustain the ability to self-renew and differentiate. These stem cell properties are tightly controlled by signaling pathways that orchestrate complex transcriptional and posttranscriptional layers of gene regulation. The structural foundation of these cell type-specific transcriptional programs is determined by DNAprotein-RNA complexes within the nuclear space. In 1879, Walther Flemming first described this complex structure in mitotic salamander cells, terming it "chromatin" from the Greek word chroma, referring to the color affinity of the intensely stained nuclear content. Almost a century later, X-ray diffraction patterns of chromatin by Maurice Wilkins, Vittorio Luzzati, and Aaron Klug suggested a repeating building unit and that histones are involved in packaging DNA (Luzzati and Nicolaieff, 1959; Wilkins et al., 1959). Indeed, subsequent enzymatic digestion of chromatin isolated from rat liver cells using DNA nuclease revealed multiples of 200 base pair DNA fragments (Hewish and Burgoyne, 1973). Electron micrographs of chromatin fibers also revealed 
that these repeating units, known as nucleosomes, were composed of DNA wrapped around histone molecules (Kornberg, 1974; Olins and Olins, 1974; Oudet et al., 1975). Two decades later, Karoline Luger's structural studies determined that the core of the nucleosome consists of 147 base pairs of DNA wrapped around an octamer of histones assembled from a tetramer of histone $\mathrm{H} 3: \mathrm{H} 4$ dimers that is flanked by two Histone H2A:H2B dimers (Luger et al., 1997). Since then, nucleosomes have become known as highly dynamic hubs of DNA-proteinRNA interactions, that not only allow for cell-type specific gene regulation, but for higher order chromatin organization important to many cellular processes.

The histones within the core nucleosome are interchangeable with different isoforms, identified as histone variants by Franklin and Zweidler (1977). While the repertoire of histones continues to expand, the Histone $\mathrm{H} 3$ family in particular has been in the spotlight of chromatin and cellular plasticity research. H3 carries the majority of well characterized heritable posttranslational modifications (PTMs) known to date, evolved a centromere specific histone variant, has a pronounced effect on nucleosome stability compared to other histones and can act as an oncogene due to mutations within critical residues subject to PTMs (Filipescu et al., 2014). Moreover, the current research on H3 shows how profound this integral nucleosome component is to the regulation of chromatin states and cell identity (Filipescu et al., 2014; Loppin and Berger, 2020; Martire and Banaszynski, 2020). The histone H3 family is composed of 8 members, H3.1, H3.2, H3.3, CENPA, H3.4, H3.5, H3.X and H3.Y. While the latter 4 members are poorly characterized, the replicative $\mathrm{H} 3.1 / \mathrm{H} 3.2$ variants and the non-replicative $\mathrm{H} 3.3$ and CENPA variants have received much attention.

H3 histone forms differ markedly in their gene structure, expression profiles, deposition mode and post translational modifications (Mendiratta et al., 2018; Martire and Banaszynski, 2020). H3.1 and H3.2 are found in multiple copies in the genome. In dividing cells, they are defined as replicative histones due to their S-phase specific expression and replication-dependent deposition, which allows for chromatin assembly in the wake of DNA synthesis when parental histones are diluted (Figure 1A; Mendiratta et al., 2018; Grover et al., 2018). The H3.3 variant differs from the H3.1 and H3.2 by only 5 and 4 amino acids, respectively. On the other hand, there are two $\mathrm{H} 3.3$ genes in mammals, $\mathrm{H} 3 \mathrm{f} 3 \mathrm{a}$ and $\mathrm{H} 3 \mathrm{f} 3 \mathrm{~b}$, that encode identical amino acid sequence but are different in their primary DNA sequence and are tightly regulated transcriptionally and post-transcriptionally in different cell types (Muhire et al., 2019). H3.3 genes are expressed throughout the cell cycle in dividing cells (Figure 1) and are highly abundant, if not the predominant $\mathrm{H} 3$, in non-dividing cells. Finally, CENPA, the centromere specific H3 variant, shares less than $51 \%$ sequence identity with the replicative histones and forms a highly compacted nucleosome core that is wrapped by only 121 base pairs of DNA. It is encoded by one gene expressed during G2 and mitosis in preparation for new CENPA incorporation in centromeres (Figure 1B; Jansen et al., 2007; Martire and Banaszynski, 2020). For recent evolutionary analysis of $\mathrm{H} 3$ variants and their role in development and disease, readers are referred to (Buschbeck and Hake, 2017; Loppin and Berger, 2020).
Overall, the positioning, modifications, and histone composition of nucleosomes can have profound effects on chromatin accessibility to transcription factors at actively transcribed (euchromatic) and repressed (heterochromatic) compartments, whose activity determines cell identity. Indeed, recent integration of different epigenomic maps, including higher order chromatin structures, nucleosome positioning, histone distribution and modifications during early development, and different cell fate change paradigms, demonstrates the complexity of spatiotemporal chromatin rearrangements (Eckersley-Maslin et al., 2018; Pérez-Palacios and Bourc'his, 2018; Fang et al., 2018; Ishiuchi et al., 2021). However, how nucleosome components and assembly pathways contribute to this regulation is still being dissected.

\section{Histone Chaperone Roles in Nucleosome Dynamics and Beyond}

Nucleosomes are diverse and dynamic. They can be shifted, assembled, or disassembled, and organized into different chromatin compartments through cooperation of histone chaperones, chromatin remodelers, and chromatin modifying factors (Dixon et al., 2012; Struhl and Segal, 2013). In particular, histone chaperones are the life partners of the nucleosome's core histones. They are involved in escorting histones from their synthesis, storage, and transport, to histone modification, deposition, eviction, and recycling in the nucleosome (Grover et al., 2018).

Originally, the term "molecular chaperone" was used by Ron Laskey who isolated and characterized the function of nucleoplasmin as the first histone chaperone using Xenopus egg extracts (Laskey et al., 1977, 1978). This was the proof of principle that histone chaperones are involved in nucleosome assembly by binding directly to histones, neutralizing their positive charges and preventing non-specific interactions and aggregates formed with DNA in vitro under physiological salt concentrations (Dilworth et al., 1987). This seminal discovery laid the groundwork for our knowledge today that nucleosome assembly involves a step wise transfer of $\mathrm{H} 3: \mathrm{H} 4$ and $\mathrm{H} 2 \mathrm{~A}: \mathrm{H} 2 \mathrm{~B}$ dimers and a complex network of histone chaperone partners (Hammond et al., 2017). As a whole, histone chaperones have no consensus sequences or structural motifs, making the discovery of novel chaperones more challenging. They exhibit considerable differences perhaps due to specialized functions in (1) recognition of distinct histone variants, (2) dedicated activities in different DNA transactions: replication, transcription, repair, and recombination, (3) diverse complex formation in histone dependent or independent manners and (4) spatiotemporal requirements in different celltypes.

Histone H3 and its variants especially exhibit a highly complex and specialized histone chaperone network in addition to more general chaperone interactions as seen with other histones (Figure 1). However, studying the interplay between the different histone chaperone pathways has been challenging to disentangle in the context of cell fate transitions. Recent technological advances combining histone labeling, genetic 


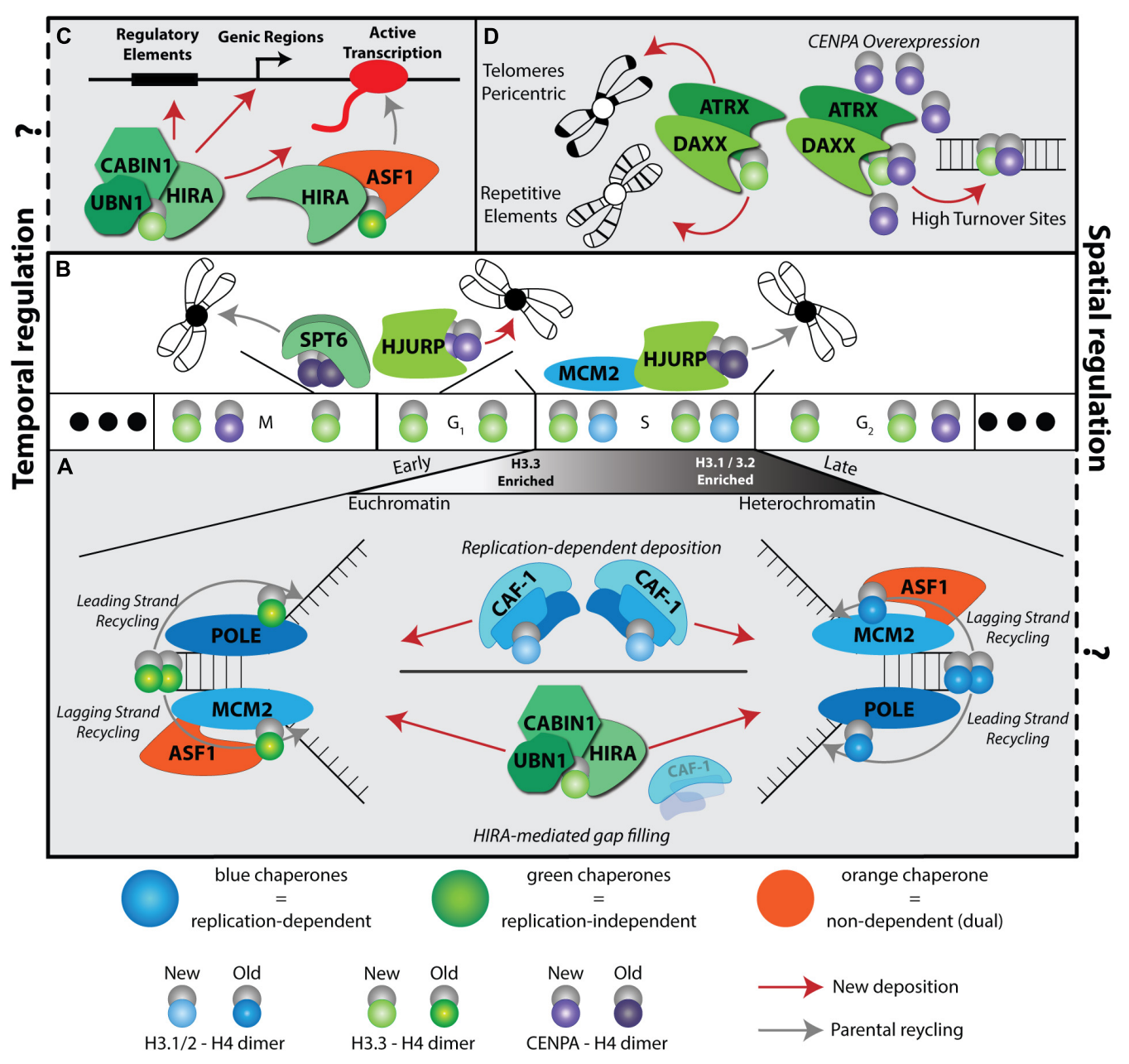

FIGURE 1 | A complex histone chaperone network regulates spatiotemporal $\mathrm{H} 3$ deposition. The temporal $\mathrm{H} 3$ expression and deposition pathways are illustrated using the cell cycle phases (G1, S, G2, M) as a centerpiece. Three solid dots on the ends indicate continuous cycling. Histones H3.1/2 (blue) are expressed during S phase, H3.3 (green) is expressed throughout the cell cycle and CENPA is expressed during G2 and mitosis. Histone deposition molecules are color coded: RD (blue), RI (green), Dual (orange). Recycling of old histones (dark shaded H3 molecules) and deposition of new histones (light shaded H3 molecules) are indicated by gray and red arrows, respectively. The spatial deposition is illustrated either in the context of heterochromatin or euchromatin compartments, transcription sites or repetitive elements. (A) CAF-1 trimer deposits new H3.1/2 during S phase. ASF1 and MCM2 cooperate to promote histone recycling to the lagging strand, while subunits POLE3-POLE4 recycle histones to the leading strand. Early replicating regions occur in euchromatin and are enriched with $\mathrm{H} 3.3$ histones, while late replicating regions occur in heterochromatic regions and are enriched with replicative histone H3.1/2. In the absence of CAF-1, HIRA deposits H3.3 at replication sites in a gap-filling mechanism. (B) CENPA is deposited in a spatiotemporal manner. HJURP deposits new CENPA at centromeres during late M and early G1 phases. SUPT6 recycles parental CENPA during transcription in late M and early $\mathrm{G} 1$ to prevent CENPA eviction at centromeres. HJURP cooperates with MCM2 during S phase to recycle parental CENPA at the centromere. (C) HIRA-mediated H3.3 deposition at a transcriptional unit. Two different HIRA complexes deposit new and parental H3.3. (D) DAXX-ATRX deposits H3.3 at telomeres, pericentric regions, and repetitive elements. When CENPA is overexpressed, DAXX-ATRX deposits heterotypic tetramers containing both $\mathrm{H} 3.3$ and CENPA at sites of high histone turnover.

engineering, epigenomics, high resolution microscopy, and structural and biochemical approaches in different contexts have started to shed light on understanding the role of nucleosome dynamics in cell fate decisions. In this review, we focus on $\mathrm{H} 3$ deposition pathways in the context of the cell cycle and how they relate to cell fate transitions during early development and several culture systems (Figures 2,3 and Table 1). The roles of other histone variants, accompanying chaperones, chromatin remodelers and modifiers in cell fate transitions are reviewed in recent publications, including this special issue.

\section{HISTONE H3 DEPOSITION PATHWAYS}

\section{Spatiotemporal Regulation of CAF-1, HIRA and DAXX Pathways}

Early work on nucleosome assembly pathways demonstrated a specificity of histone $\mathrm{H} 3$ chaperones to assemble nucleosomes in a DNA replication dependent (RD) or independent (RI) manner (Almouzni and Méchali, 1988; Smith and Stillman, 1989; Ray-Gallet et al., 2002). This cell cycle determinant of histone chaperone pathways is further complicated by the specific 
partnerships of histone chaperones with different variants, parental "old" histones versus newly synthesized histones and the deposition coordinates in the genome (Figure 1). Assembly of newly synthesized histones has been extensively studied and recent work is beginning to uncover the recycling mechanisms of old histones (Venkatesh and Workman, 2015; Serra-Cardona and Zhang, 2018). Moreover, some plasticity and redundancy across these pathways has been observed, especially in terms of handing over new histones, recycling old histones, or when some pathways are absent or compromised (placeholding/gap filling) (Figures 1A,B) (Dunleavy et al., 2011; Ray-Gallet et al., 2011; Schneiderman et al., 2012).

The first discovery of a RD histone chaperone was reported more than three decades ago from human cell extracts by the purification and characterization of the Chromatin Assembly Factor complex CAF-1, a trimeric subunit composed of p150, p60 and Rbbp4 (Smith and Stillman, 1989). To date, CAF-1 is the only known histone chaperone that loads newly synthesized H3.1/2:H4 dimers onto DNA in a RD manner (Figure 1A).

It took another decade to discover the Histone H3.3-specific chaperone HIRA (histone cell cycle regulator). Subsequently, HIRA was found to also function as a trimeric complex with UBN1 and CABIN subunits (Lorain et al., 1998; Magnaghi et al., 1998). The HIRA complex incorporates newly synthesized H3.3 in a RI manner and was initially associated with histone deposition at active sites of transcription (Ahmad and Henikoff, 2002; Ray-Gallet et al., 2002; Figure 1C).

Genome wide distribution of $\mathrm{H} 3.3$ deposition in mouse embryonic stem cells (mESCs) led to the discovery of an additional H3.3-specific histone chaperone death domain associated protein (DAXX) (Tang et al., 2004; Goldberg et al., 2010; Lewis et al., 2010). DAXX in complex with the chromatin remodeler ATRX specializes in the deposition of $\mathrm{H} 3.3$ at repetitive elements, including telomeres, pericentromeric DNA, and a subset of endogenous retroviral elements (Goldberg et al., 2010; Lewis et al., 2010; Elsässer et al., 2015; Hoelper et al., 2017; Figure 1D). Although, the cell cycle timing of DAXX mediated H3.3 deposition is unclear, considering that the deposition of newly synthesized CENPA on centromeres via the Holliday junction recognition protein (HJURP) histone chaperone occurs in late mitosis/early G1 phase (Dunleavy et al., 2009; Figure 1B), it is tempting to speculate that the $\mathrm{H} 3.3$ deposition at repetitive elements coincides with this timing.

During $S$ phase, HJURP mediates parental CENPA recycling with MCM2, a subunit of the helicase complex, and H3 histones act as place holders until new CENPA is deposited (Dunleavy et al., 2011; Zasadziñska et al., 2018; Figure 1B). Notably, CENPA overexpression leads to heterotypic deposition with $\mathrm{H} 3.3$ by the histone chaperone DAXX, reinforcing the plasticity of the histone chaperone network (Figure 1D; Arimura et al., 2014; Lacoste et al., 2014).

\section{The ASF1 Hub}

The ASF1 histone chaperone, originally discovered in yeast (Tyler et al., 1999), serves dual RD and RI nucleosome assembly pathways as it functions in handing over newly synthesized
H3.1:H4 and H3.3:H4 dimers to CAF-1 and HIRA complexes, respectively (Grover et al., 2018). In mammals, the ASF1 network is diversified by the emergence of two paralogs, ASF1A and ASF1B, with preferences for HIRA and CAF-1 p60, respectively (Tang et al., 2006; Abascal et al., 2013). Interestingly, recent work by Almouzni's group demonstrated that ASF1 coordinates with HIRA to distinguish between old and new histone incorporation during transcription. In this model, ASF1 participates in the HIRA complex to recycle old H3.3, while new H3.3 is deposited via a UBN1-containing complex (Figure 1C; Torné et al., 2020).

Depletion of both ASF1 paralogs showed ASF1 is important for histone recycling during replication. This recycling is accomplished in partnership with MCM2, a subunit of the helicase complex. Together, they promote the recycling of old H3:H4 dimers in a RD manner (Groth et al., 2007; Huang et al., 2015; Figure 1A). Furthermore, recent evidence indicates MCM2 promotes symmetric loading of parental H3:H4 during DNA replication by preventing biased parental histone loading on the leading strand (Petryk et al., 2018). Conversely, newly identified histone chaperones POLE3POLE4, subunits of the leading strand polymerase POLE, are proposed to load parental $\mathrm{H} 3: \mathrm{H} 4$ on the leading strand (Bellelli et al., 2018; Figure 1A). Considering these intriguing findings, it will be interesting to probe the interplay between POLE subunits, MCM2, and ASF1 and how leading and lagging strand deposition is balanced during self-renewal or differentiation.

Genome wide distribution and high-resolution microscopy mapping of parental histones in human cells demonstrate that $\mathrm{H} 3.1$ and $\mathrm{H} 3.3$ associate with late and early replicating regions, respectively (Clément et al., 2018; Mendiratta et al., 2018; Figure 1A). Therefore, it is tempting to hypothesize that due to its preference for HIRA interaction, ASF1A could act by recycling $\mathrm{H} 3.3$ while ASF1B could participate in recycling $\mathrm{H} 3.1 / 2: \mathrm{H} 4$ at the replication fork. It would therefore be interesting to investigate how ASF1 paralogs could participate in loading parental H3.1/2:H4 and H3.3:H4 dimers depending on their associated partners, replication sites/timing and leading versus lagging strand preference.

Another histone chaperone discovered in yeast, Suppressor of Ty 6 (SPT6) (Kaplan et al., 2003) plays a role in recycling parental $\mathrm{H} 3: \mathrm{H} 4$ during transcription and ASF1 can fill in the nucleosome gaps in spt6 yeast mutants (Jeronimo et al., 2019). Interestingly, yeast has only one form of $\mathrm{H} 3$ that is closely related to the mammalian H3.3 variant (Talbert and Henikoff, 2010). Given that SPT6 has an important role in transcription elongation (Vos et al., 2018, 2020), the interplay of ASF1 and SPT6 in histone deposition and recycling during transcription may yield further insights into nucleosome dynamics during this process. While commonly cited as a $\mathrm{H} 3$ histone chaperone, as shown in yeast, it remains to be concretely determined if SPT6 functions in H3:H4 deposition in mammalian cells. However, in support of this hypothesis, a recent study proposes a role for the histone chaperone SPT6 as a recycling factor for CENPA, with evidence in Drosophila and HeLa cells (Bobkov et al., 2020; Figure 1B). 
Altogether the recent advances in labeling and mapping spatiotemporal distribution of old and new histones, structural mechanisms of histone-chaperone recognition, how they interact directly with DNA templates, and mapping nucleosome positions during different DNA processes have deepened our understanding on how the cell uses nucleosome assembly to maintain or reprogram chromatin organization. While mechanistic studies are largely performed in vitro or in immortal human or mouse cell lines, this knowledge could provide the fundamental mechanisms at play for stem cell maintenance and lineage commitment during development and tissue homeostasis (see sections below).

\section{H3 DEPOSITION IN DIFFERENT PHYSIOLOGICAL SETTINGS}

How the nucleosome assembly pathways discussed above (Figure 1) modulate cell fate decisions and cell identity maintenance puzzled scientists for decades. The recent breakthroughs in the field coincide with a burst of technological advances and their relevant applications in studying culture models and developmental processes (Figure 2). A wide spectrum of phenotypes has been observed upon manipulation of RD and RI nucleosome assembly pathways that depend on spatiotemporal histone requirements, with $\mathrm{RD}$ pathways arguably more challenging to study due to their requirement in cellular proliferation and subsequent lethality. Here, we will describe some examples and discuss possible mechanisms along with future implications.

\section{CULTURE MODELS}

Culture models to study cellular differentiation and reprogramming are powerful platforms to explore the molecular mechanisms orchestrated by the histone variants-histone chaperone network because they provide an opportunity to study cell autonomous effects within specific lineages, with some systems being more homogenous compared to others and are compatible with biochemical approaches (Figure 2A). Here we describe stem cell-based systems that mimic normal development and that have proved useful in understanding H3 deposition pathways.

\section{Embryonic Stem Cells}

ESCs are the earliest embryonic cells that can be captured in vitro from the blastocyst and propagated without compromising their pluripotent potential (Figure 2A; Evans and Kaufman, 1981; Martin, 1981). A change in culture conditions and/or intrinsic factors can coax ESCs to interchange their potency levels and/or commit to different lineages (Figure 2A). For example, ESCs can be maintained in culture in various states of pluripotency reflecting naïve (ground) or primed developmental states. Human ESCs (hESCs) derived from blastocysts reflect an even later pluripotency state during mouse development akin to the epiblast stem cells (epiSCs) that can be derived from the post implantation mouse embryo (Figure 2A).

In contrast to the developmental arrests that have been observed in CAF-1 depleted embryos (Houlard et al., 2006), it has been more amenable to probe the function of $\mathrm{H} 3$ deposition pathways particularly in mESCs without compromising cellular viability. The loss of the Chafla and Chaf1b subunits of the CAF-1 complex in ESCs results in their reprogramming to an earlier embryonic cell state mimicking the two-cell stage of embryonic development (2C-like cells) (Ishiuchi et al., 2015, 2021). The conversion of ESCs to 2C-like cells is dependent on cell progression through S-phase and on the chromatin assembly activity of CAF-1, displaying similar molecular features to spontaneously derived 2C-like cells and 2-cell stage embryos. Although, the recent finding that $\mathrm{H} 3.3$ deposition resumes non-canonical distribution upon CAF-1 ablation in ESCs suggests that this 2C-like cell fate induction is in fact reflecting a transient or even earlier embryonic cell state (Ishiuchi et al., 2021; Table 1). It will be interesting to compare additional epigenetic features and transcriptomes during this developmental window in the CAF1 mutants.

This induction of a permissive chromatin state upon CAF-1 loss in ESCs is consistent with the initial observations where CAF-1 loss in ESCs affects heterochromatin features specific to stem cells (Houlard et al., 2006). Indeed, recent work demonstrated that CAF-1 loss impairs ESC differentiation in an embryoid body assay (Cheng et al., 2019; Figure 2A). Interestingly, this defect was linked to failure of establishing $\mathrm{H} 3 \mathrm{~K} 27 \mathrm{me} 3$ marks at pluripotency promoters upon differentiation through CAF-1-PCNA and CAF-1-Polycomb (PRC2) recruitment to the replication fork. In this context, Cheng et al. (2019) detected a reduced association of H3.1 and H3K27me3 with replicating chromatin. It would be interesting to test how $\mathrm{H} 3.1 / \mathrm{H} 3.3$ ratio affects $\mathrm{H} 3 \mathrm{~K} 27$ me3 establishment and how parental histone inheritance is influenced during this process, potentially conferring a resistance of CAF-1 ESCs to differentiation.

Parental and new histone distribution was examined at a single cell level using a Wnt3a-induced asymmetric ESC division model, demonstrating there is differential distribution of old and new canonical histones in the daughter cells (Ma et al., 2020). This suggests a specialized action of histone chaperones during asymmetric division. Additionally, in light of the new implication that MCM2 promotes symmetric cell division through RD histone recycling to the lagging strand (Bellelli et al., 2018; Petryk et al., 2018), possibly with ASF1 as seen in HeLa cells (Bellelli et al., 2018; Petryk et al., 2018; Figure 1A), it will be interesting to examine how perturbation of different histone chaperones in ESC self-renewal and differentiation affects histone distribution during replication. Of note, the involvement of CAF-1 and histone mutations has been previously proposed to play a role in asymmetric histone deposition during C. elegans development (Nakano et al., 2011). Whether similar mechanisms are conserved in mammals remains to be tested.

Contrary to the loss of CAF-1 in ESCs, perturbation of RI nucleosome assembly in ESCs does not alter gene expression 
profiles nor compromise ESC identity under self-renewal conditions. However, H3.3 together with its partners DAXX and ATRX are involved in silencing repetitive elements in ESCs, including a subset of retroelements and telomeres (Goldberg et al., 2010; Lewis et al., 2010; Elsässer et al., 2015; Hoelper et al., 2017; Figure 1D). Remarkably, this effect is more pronounced in more naïve or hypomethylated ESC cultures reflecting an important role in early preimplantation development (He et al., 2015). Whether this effect is purely a function in safeguarding genome stability or fine tuning transcriptional programs coopted by repetitive elements remains to be explored (Macfarlan et al., 2012). The physiological effect of RI nucleosome assembly pathway depletion is exacerbated upon differentiation of ESCs where lineage specific gene expression programs are perturbed.

Interestingly the loss of ASF1A, HIRA and H3.3 affect Histone H3 K27 methylation (H3K27me3) specifically at developmentally regulated genes (Banaszynski et al., 2013; Gehre et al., 2020; Gao et al., 2018; Figure 2A and Table 1). HIRA dependent deposition of $\mathrm{H} 3.3$ is proposed to establish bivalent marks in ESCs at developmentally regulated genes while ASF1A dependent disassembly of nucleosomes facilitates resolution of bivalent domains upon ESC differentiation. H3.3 loss in ESCs also reduces enhancer $\mathrm{H} 3$ acetylation marks including H3K27ac, H3K18ac, H3K64ac, and H3K122ac (Martire et al., 2019). H3K27ac, in particular, a mark known to coincide with active enhancers is stimulated by the phosphorylation of the serine 31 residue on the H3.3 tail in mESCs. H3.3 serine 31 (H3.3S31) is one of the amino acids unique to H3.3. Supplementing H3.3 KO mESCs with replicative histone H3.2, bearing an alanine 31 residue, cannot rescue the enhancer acetylation defect despite being deposited at these sites. Moreover, the loss of H3.3 in ESCs does not affect chromatin accessibility or the recruitment of p300 histone acetyltransferase at enhancer elements suggesting that the $\mathrm{H} 3.3 \mathrm{~S} 31$ residue is uniquely required downstream of HIRA mediated deposition for subsequent chromatin signaling pathways. Consistent with the loss of H3K27me3 or DNA methylation in ESCs, the reduced acetylation of the H3.3 $\mathrm{KO}$ is tolerated by ESCs under self-renewing conditions with no dramatic effect on gene expression (Martire et al., 2019). However, their differentiation triggers defects in chromatin accessibility and establishing active enhancer elements and subsequent activation of differentiation genes.

A recent systematic characterization of all four H3.3 specific residues in a Xenopus gastrulation model reinforces the essential role of $\mathrm{H} 3.3 \mathrm{~S} 31$ specific phosphorylation during this developmental process (Sitbon et al., 2020). Strikingly, the replacement of all three $\mathrm{H} 3.3$ residues that are required for specific RI chaperone interactions with their $\mathrm{H} 3.2$ replicative counterparts was compatible with normal gastrulation. It will be interesting to perform similar genetic analyses in the context of ESC differentiation.

Recent work interrogated the function of $\mathrm{H} 3.3$ lysine residues (K4 and K36) in ESCs (Gehre et al., 2020). Alanine substitutions of $\mathrm{H} 3.3 \mathrm{~K} 4$ and $\mathrm{H} 3.3 \mathrm{~K} 36$ did not compromise ESC self-renewal but perturbed lineage specific transcriptional programs and differentiation, albeit with varying degrees. H3.3K4, but not H3.3K36, mutant ESCs exhibited severe defects and resulted in reduced $\mathrm{H} 3.3$ deposition at regulatory elements, especially promoters, independently of the lysine charge. While wild type replicative histones share these same residues with $\mathrm{H} 3.3$ and are able to compensate and maintain normal nucleosome density around transcription start sites (TSS), this is not sufficient to maintain the correct chromatin state. This observation reinforces the importance of $\mathrm{H} 3.3$ specific residues. Interestingly, $\mathrm{H} 3.3 \mathrm{~K} 4$ mutation did not perturb H3.3 histone chaperone expression or binding but diminished the interactions with chromatin remodelers and increased RNA polymerase activity. The authors thus propose a role for $\mathrm{K} 4$ in maintaining $\mathrm{H} 3.3$ at regulatory elements through proper recruitment of remodelers and accurate transcriptional activity. This study highlights how histone chaperones act in concert with remodelers and accompanying PTM signals to regulate nucleosome dynamics.

Taken together, these H3.3 studies in ESCs and model organisms justify some of the needs to incorporate $\mathrm{H} 3.3$ at regulatory elements and highlight the relevance of unique and common $\mathrm{H} 3$ residues in regulating nucleosome dynamics and setting specialized chromatin environments post nucleosome assembly (Figure 2 and Table 1).

Considering these findings, it is tempting to speculate that during mESC differentiation, $\mathrm{RD}$ assembly pathways play a passive role in diluting ESC identity and RI pathways play an active role in establishing new identity. However, discrepancies in the effect of manipulating these pathways between hESCs compared to mESCs still need to be resolved. For example, the loss of both HIRA and ASF1 compromise hESCs self-renewal (Gonzalez-Muñoz et al., 2014; Zhu et al., 2017). HIRA loss in hESCs results in downregulation of pluripotency factors, activation of various lineage markers and differentiation. Moreover, in hESCs, the HIRA complex is proposed to associate with a stem cell specific subunit PROHIBITIN that stabilizes distinct complexes and cooperates with HIRA to regulate the metabolic circuitry in hESCs through H3.3 deposition. Considering that hESCs resemble mouse epiSCs (Figure 2A), which reflect a more primed pluripotent cell state, it is possible that phenotypes similar to mESCs could arise when examined in more naïve hESCs (Brumbaugh et al., 2019). It would be exciting to probe histone exchange dynamics and histone chaperone networks during interconversion of these pluripotency states to build on the current study documenting the changes in histone modifications to shape chromatin environments (De Clerck et al., 2019).

\section{Reprogramming and Transdifferentiation}

Reprogramming and transdifferentiation platforms have proved valuable in revealing unprecedented physiological roles of nucleosome assembly pathways in somatic cells (Figure 2A). For example, probing the function of CAF-1 in the context of transcription factor mediated reprogramming of mouse embryonic fibroblasts to induced pluripotent stem cells (iPSCs) implicated its role in maintaining somatic cell identity. In this system, CAF-1 is proposed to act in part through its nucleosome assembly function by restricting access to pluripotency transcription factors (Cheloufi et al., 2015; 
Cheloufi and Hochedlinger, 2017). Supporting the role of CAF-1 in reprogramming, CAF-1 depletion in mESCs facilitates the generation of cloned blastocysts using somatic cell nuclear transfer technology and transdifferentiation between different lineages (Cheloufi et al., 2015; Ishiuchi et al., 2015). Contrary to CAF-1, ASF1A loss inhibits reprogramming of human somatic cells to iPSCs (Gonzalez-Muñoz et al., 2014). In this system, ASF1A co-expression with pluripotent transcription factor OCT4 is sufficient to reprogram human adult dermal fibroblasts when exposed to the oocyte-specific paracrine growth factor GDF9. In this context, ASF1A is proposed to work by promoting acetylation of histone $\mathrm{H} 3 \mathrm{~K} 56$ and cooperating with OCT4 to activate the pluripotency transcriptional network. ASF1A acts upstream of CAF-1 as a donor of newly synthesized histones but its functions also overlap with other nucleosome assembly pathways (Figures 1A,C). Thus, this discrepancy in reprogramming phenotypes between CAF-1 and ASF1A can be purely dependent on the spatiotemporal requirement of histone deposition and/or histone chaperone-independent functions. The implication of ASF1A in cellular reprogramming stemmed from it being a maternally deposited factor in the oocyte cytoplasm. Similarly, the H3.3 histone variant proved to be an essential maternal factor for reprogramming and the development of fertilized, parthenogenetically derived and SCNT embryos (Wen D. et al., 2014) (Figure 2A). In this context, H3.3 plays an important role in nucleosome remodeling in either the parental pronuclei or the donor nucleus (Figure 2A). Consistent with a spatiotemporal requirement of histone deposition pathways in shaping cellular identity, a recent study demonstrates a dual role of HIRA mediated H3.3 deposition in maintaining somatic cell identity and establishing pluripotency during reprogramming (Fang et al., 2018). Thus, this global rearrangement of $\mathrm{H} 3.3$ deposition akin to the one observed during oogenesis and the early cleavage embryo represents an important mechanism in preparation for cell fate conversions (see preimplantation development \& Ishiuchi et al., 2021). However, the interplay with other histone chaperone pathways remains to be determined especially in a setting where the cell cycle is required for cell fate switches.

In light of these observations, we propose that nucleosome pathways at different potency states during development can dictate cell identity maintenance versus cell fate commitment or reprogramming toward different lineages. This could be purely dependent on specific remodeling of histone variants distribution and cell cycle properties (Figure 3 ).

\section{FROM GAMETOGENESIS TO EARLY EMBRYONIC DEVELOPMENT}

\section{Gametogenesis}

The sperm and oocyte are highly specialized cell types that transmit both genetic and epigenetic information through generations (Figure 2B). During spermatogenesis, the genome undergoes a stepwise replacement of histones with transition proteins and ultimately protamines to form the highly condensed nucleus of the sperm (Raja and Renkawitz-Pohl, 2005; TorresFlores and Hernández-Hernández, 2020). This process is thought to prevent DNA damage, confer better sperm quality, and reprogram the paternal nucleosomes in preparation for fertilization as protamine knockouts result in defective sperm and developmental arrest (Cho et al., 2001, 2003). The nuclear condensation within the sperm head is accompanied with complex PTMs of the disassembled histones and the newly deposited protamines which could potentially involve the action of different histone chaperones whose identity remains to be determined. However, despite the removal of nearly $90 \%$ of all histones in the sperm, CENPA is retained. Also, select nucleosomes at regulatory DNA elements retain $\mathrm{H} 3.1 / 2$ and H3.3 (Hammoud et al., 2009; Erkek et al., 2013; Das et al., 2017). The retention of nucleosomes containing specific histone variants and corresponding PTMs on the paternal genome is thought to be a mechanism for transmitting epigenetic information to the embryo (Champroux et al., 2018). Of note, profiling the accurate histone distribution in the sperm nucleus has proved to be technically challenging depending on the method used to purify mature sperm that have undergone proper histone replacement and chromatin digestion for histone pull downs (Yoshida et al., 2018).

Consistent with the histone retention in the mature sperm, genetic studies support these observations. To date, several mouse knockout and conditional alleles of the two H3.3 genes have been generated albeit with variable phenotypic consequences on the germline and embryonic development (see post-implantation development) possibly due to the different targeting strategies, genetic heterogeneity of the mouse strains as well as possible redundancy with testis specific $\mathrm{H} 3$ variants. For example, in a mixed C57BL/6 and 129 mouse background, $\mathrm{H} 3 \mathrm{f} 3 \mathrm{a}+/-$; $\mathrm{H} 3 \mathrm{f} 3 \mathrm{~b}-/-$ compound mutant with one remaining copy of the H3f3a gene are male sterile (Jang et al., 2015) while other studies reported that the surviving single $\mathrm{H} 3 \mathrm{f} 3 \mathrm{a}$ and H3f3b knockouts have variable levels of sterility (Couldrey et al., 1999; Bush et al., 2013; Tang et al., 2013, 2015; Yuen et al., 2014). Regardless of these differences, accumulating evidence supports a unique role of $\mathrm{H} 3.3$ in chromatin remodeling in the male germline.

The effect of H3.3 loss in the female germline is more debatable. In contrast to previous studies reporting female sterility of single $\mathrm{H} 3.3$ knockouts, $\mathrm{H} 3 \mathrm{f} 3 \mathrm{a}+/-$; $\mathrm{H} 3 \mathrm{f} 3 \mathrm{~b}-/-$ compound mutant females are viable and fertile (Bush et al., 2013; Jang et al., 2015; Tang et al., 2013, 2015; Figure 2B). This is surprising given that mature oocytes are devoid of replicative histones in their genome and that there is H3.3 redistribution during oogenesis in preparation for embryogenesis (Ishiuchi et al., 2021) (see preimplantation development). Furthermore, the requirement of $\mathrm{H} 3.3$ histone partners during gametogenesis warrant further investigations. Interestingly, Asflb knockout mice are viable but have reduced reproductive capacity showing a more severe defect in females versus males (Messiaen et al., 2016). This study showed that ASF1B is specifically expressed in the female gonads during development and propose its role in regulating meiotic entry. In light of these findings and the proposed molecular function of ASF1 (see The ASF1 Hub), it is 


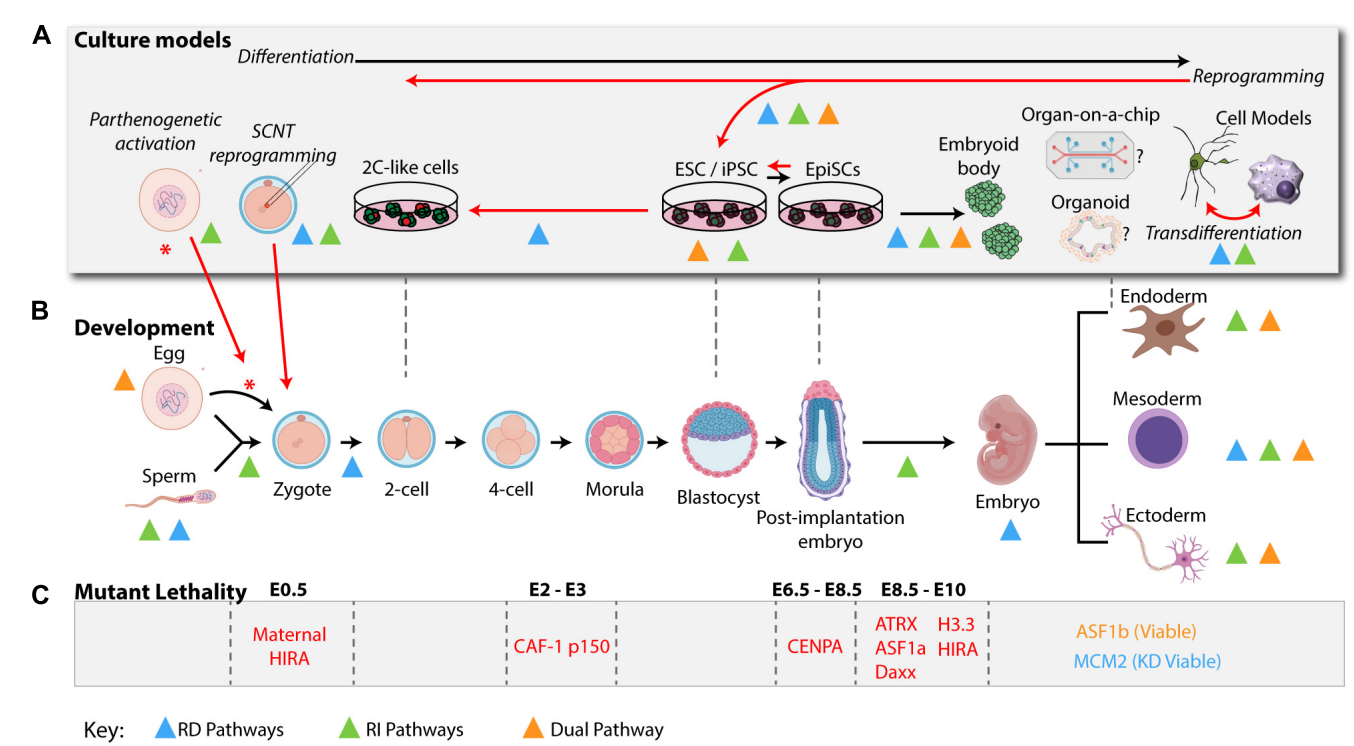

FIGURE 2 | H3 deposition pathways in a physiological setting: development and culture models. H3 Deposition pathways characterized in cell fate transitions during development or in cellular systems are indicated by colored triangles using the same color scheme used in Figure $\mathbf{1}$ (RD: blue, Rl: green, and dual: orange). (A) Ex vivo models are represented above their most relevant developmental stages as indicated in the developmental timeline (B) Black arrows indicate differentiation of the zygote from a higher potency to a lower potency. Red arrows indicate key cell plasticity pathways, including, zygote reprogramming following oocyte activation via parthenogenesis or SCNT, reprogramming of somatic cells to pluripotency and transdifferentiation of cells directly from one lineage to another. (B) A mouse developmental timeline, depicting the sperm and oocyte generating the zygote, early cleavage embryos, blastocyst, and early post-implantation embryo followed by specialized lineages discussed in the text (mouse development icons were created using BioRender software). (C) Summary of histone variants or histone chaperone mutant lethality in early embryo development. See Table $\mathbf{1}$ for a summary of phenotypes and corresponding references.

tempting to speculate that the Asflb paralog plays a specialized role in retaining $\mathrm{H} 3.3$ containing nucleosomes in a $\mathrm{RD}$ manner in the oocytes. On the other hand, given that it does not have an effect on the male germline, it might function independent of its histone chaperone role.

Notably, due to the early lethality of most histone chaperone mutants (see Preimplantation Development), the use of conditional knockouts and the development of ex vivo gametogenesis culture systems (Hamazaki et al., 2021) will be instrumental in resolving these limitations. Furthermore, this will shed light on the mechanisms and differences in spatiotemporal $\mathrm{H} 3$ re-distribution during spermatogenesis and oogenesis and how germline reprogramming may impact epigenetic inheritance.

\section{Preimplantation Development}

The fertilization between the sperm and oocyte gives rise to the zygote. In the zygote, both the paternal and maternal genomes undergo dramatic reprogramming events to give rise to the most plastic embryonic cell state known as "totipotency" (Figures 2A,B). If successful, the zygote will ultimately give rise to all cell types necessary for the development of an organism including the extraembryonic tissues. During this process, both the paternal and maternal pronuclei undergo major chromatin remodeling using maternally deposited factors in preparation for the first mitotic divisions and the transition to zygotic transcription (Figure 2B; Probst and Almouzni, 2011; EckersleyMaslin et al., 2018). Accumulating evidence supports the idea that maternally deposited histones and histone chaperones are essential for reprogramming the zygote following fertilization. Indeed, the paternal genome is decondensed when incorporation of maternally deposited H3.3 replaces protamines, allowing for genome reprogramming (Loppin et al., 2005; Torres-Padilla et al., 2006). This is now known to be triggered by site specific phosphorylation of protamines by the RNA splicing factor SRPK1 which permits recruitment of nucleoplasmin (NPM2) and HIRA for protamine unloading and H3.3 deposition, respectively (Gou et al., 2020).

The manipulation of maternally deposited factors in oocytes followed by natural fertilization, parthenogenetic activation, or somatic cell nuclear transfer has been instrumental in understanding the mechanisms of RI incorporation of $\mathrm{H} 3.3$ onto parental chromatin (Figure 2B and Table 1; Lin et al., 2014; Wen D. et al., 2014). For example, deletion of HIRA in mouse oocytes results in inhibition of nucleosome assembly in the male genome and oocytes are unable to develop parthenogenetically. This study links HIRA-dependent H3.3 deposition to active transcription of ribosomal RNA in the zygote (Lin et al., 2014).

It will be interesting to probe the function of other H3.3 mediated site-specific histone chaperone pathways in the oocytes. Using ultra-low input native CHIP-seq a recent study generated a spatiotemporal map of H3.3 distribution during oogenesis, the zygote, and the early cleavage stage embryos (Ishiuchi et al., 2021). Interestingly, H3.3 undergoes a gradual global rearrangement during oogenesis forming a unique non-canonical pattern in the mature oocyte and zygote. At this developmental 
window, H3.3 is more broadly distributed across the genome and exhibits some enrichment at heterochromatic regions. Remarkably, the non-canonical H3.3 distribution is similar between the maternal and paternal pronuclei in the zygote but is different from other post-mitotic cells, such as neurons. Interestingly, this unique chromatin incorporation of $\mathrm{H} 3.3$ in the oocyte and zygote coincides with previously reported distinct epigenetic features, including chromatin accessibility, histone marks and DNA methylation during preimplantation development (Eckersley-Maslin et al., 2018; Burton et al., 2020). For example, temporal regulation of histone methyltransferases (SUV39H1\&H2) involved in the deposition of the H3K9me3 repressive mark post fertilization results in establishing an accessible and non-repressive constitutive heterochromatin in the zygote that ultimately matures in later stages to a compacted and repressive state. While Ishiuchi et al., 2021 did not report a correlation between $\mathrm{H} 3.3$ deposition and $\mathrm{H} 3 \mathrm{~K} 9 \mathrm{me} 3$ profiles in the zygote, it is tempting to speculate that this early marking of heterochromatin is established as a consequence of the slight preferential loading of $\mathrm{H} 3.3$ on heterochromatin compartments in the zygote. H3.3 deposition within these domains could create a chromatin environment to recruit SUV39H1 similar to the mechanism proposed for PRC2 recruitment in ESCs at developmentally regulated genes (Figure $\mathbf{2}$ and Table 1; Banaszynski et al., 2013). However, comparison of existing ChIPseq data over constitutive heterochromatin domains may be challenging due to variable chromatin fragmentations, timing of the embryos, and considering multi-mapping reads at repetitive elements. Notably, as the zygote transitions to the 2-cell stage, the broad H3.3 distribution is reprogrammed to a more localized pattern reminiscent of the known canonical pattern initially described in ESCs. The reorganization and/or retention of $\mathrm{H} 3.3$ in the zygote occurs with the loading of replicative $\mathrm{H} 3.1 \& \mathrm{H} 3.2$ in a $\mathrm{RD}$ and transcription independent manner. Furthermore, it is regulated by CAF-1 as injecting a dominant negative form of the CAF-1 p150 subunit in the zygote reduces the canonical H3.3 rearrangement. Importantly this unique $\mathrm{H} 3.3$ rearrangement and deposition of replicative histones is essential for development as inhibition of maternally deposited CAF-1 results in developmental arrest at the 4-cell stage, consistent with previous reports ablating CAF-1 in the embryo (Figure $2 \mathrm{C}$ and Table 1; Houlard et al., 2006; Akiyama et al., 2011).

Considering the oocyte is devoid of replicative histones and that the expression of $\mathrm{H} 3.1 / 2$ peaks only after the embryo has undergone one cell division, it will be interesting to investigate how the non-canonical H3.3 distribution is propagated during the first round of replication, and how its recycling is regulated by histone chaperones in the context of replication timing. Therefore, it will be important to further characterize the histone chaperones required for H3.3 nucleosome exchange during these early cell divisions and how they are involved in preparing for zygotic genome activation and the establishment of heterochromatin and euchromatin domains. We think that the H3.3 broad distribution in the oocytes and zygote is possibly pre-programmed because of the need for fast RI eviction of protamines genome wide in the paternal pronucleus to ensure near-equal reprogramming of parental pronuclei in the zygote.

\section{Post-implantation Development}

In contrast to the severity of maternal or zygotic CAF-1 loss, mice lacking ASF1A survive to mid-gestation (Figure 2C; Hartford et al., 2011). Given that Asf1b knockout mice are viable, it would be interesting to probe phenotypic consequences of Asfla/b double knockouts. Similarly, in mice lacking either H3.3 gene and their histone chaperone partners, HIRA or DAAX, embryos also progress to mid-gestation (Figure 2C; Michaelson et al., 1999; Roberts et al., 2002; Jang et al., 2015). Interestingly, $\mathrm{H} 3 \mathrm{f} 3 \mathrm{a} / \mathrm{b}$ double knockout mice progress through early patterning of the embryo but are lethal 2 days after implantation (Figure 2C). Single knockouts of H3f3a and H3f3b, however, are reported with a spectrum of phenotypes depending on the study resulting in compromised viability and sterility (see gametogenesis). Similarly, CENP-A knockout mice are also lethal shortly after implantation (Howman et al., 2000). Of note, chromatin defects in these different mutants suggest a convergent mechanism between $\mathrm{RD}$ and $\mathrm{RI}$ pathways where heterochromatin structures are primarily affected leading to mitotic defects and developmental arrest. The relatively late phenotypic manifestations of RI nucleosome assembly pathways could be due to maternal deposition of mRNA and proteins of histones and histone chaperones, or redundancy of histone chaperone pathways. It would therefore be interesting to test the effects of their maternal contribution and the consequences of individual histone chaperone perturbation on histone deposition and chromatin accessibility in the early embryo.

While examining histone exchange and chromatin dynamics in the early embryo remain challenging, current developments in CRISPR CAS9 gene editing (Adli, 2018; Anzalone et al., 2020), chromatin profiling technologies such as ATAC-seq, CUT\&RUN and CUT\&Tag that rely on a small number of cells (Buenrostro et al., 2013; Corces et al., 2017; Kaya-Okur et al., 2019; Meers et al., 2019), single cell multi-omics (Pérez-Palacios and Bourc'his, 2018), and culture systems are instrumental in understanding the mechanisms of nucleosome assembly during these most plastic cell states during development.

\section{LINEAGE SPECIFIC DIFFERENTIATION, A MIX OF IN VIVO AND CULTURE SYSTEMS}

Given the early lethal phenotypes of the $\mathrm{H} 3$ deposition pathways during development (Figures 2B,C), it has been challenging to probe their function in normal homeostasis. However, recent work using lineage specific differentiation systems as well as cancer and injury models (Evano et al., 2020) has not only shed light on how some mechanisms described above (Figure 1) are at play, but also associates histone chaperones with histone deposition independent functions. We postulate that these differences could be intimately linked to the spatiotemporal expression of the $\mathrm{H} 3$ deposition machinery and the specific cell cycle properties (e.g.: short versus long) within different lineages (Figure 3). Evidence so far suggests an important role of both $\mathrm{RD}$ and RI pathways in lineage restriction and maintenance. For 
TABLE 1 | Histone and histone chaperone roles in cell fate decisions.

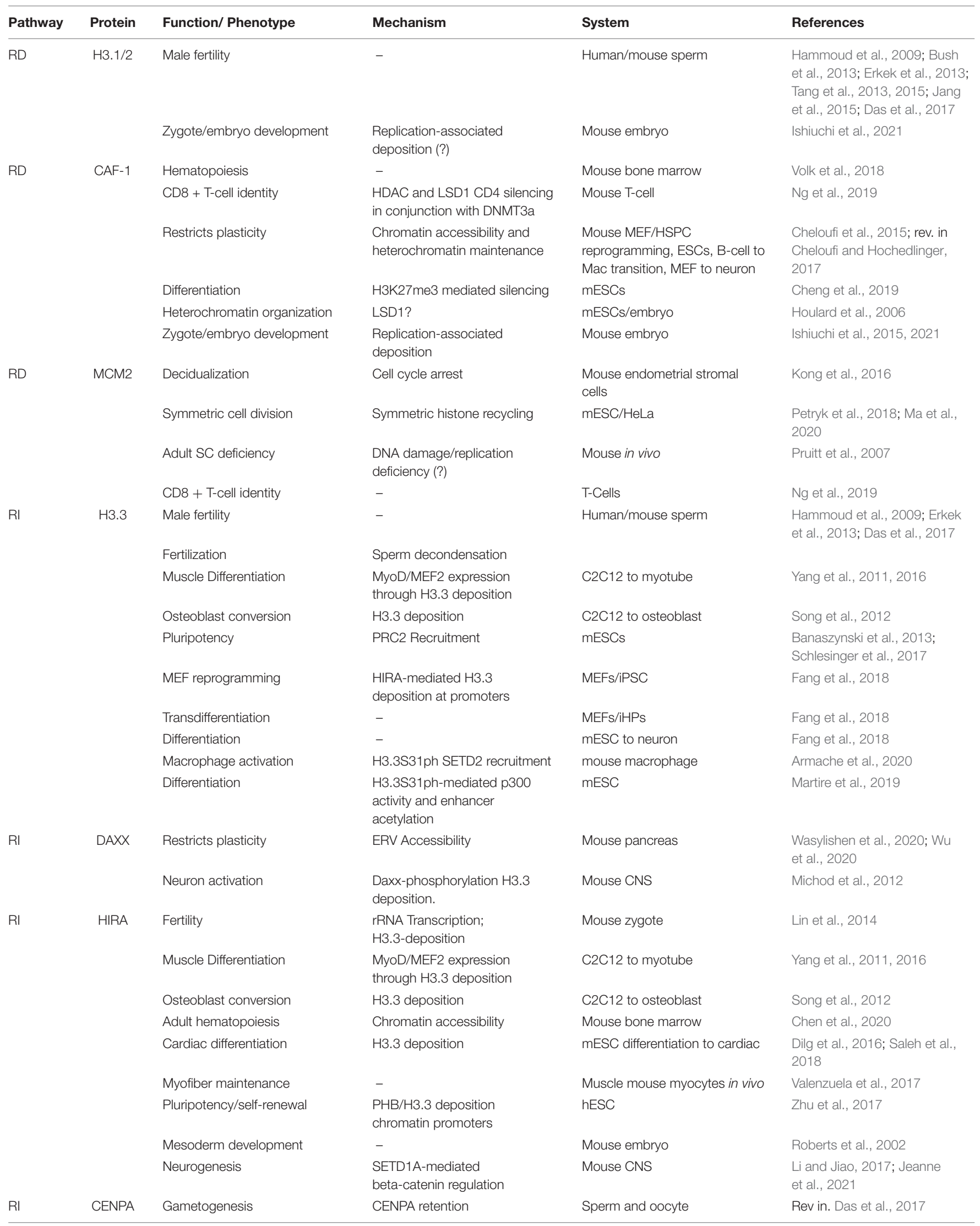


TABLE 1 | Continued

\begin{tabular}{llllll}
\hline Pathway & Protein & Function/ Phenotype & Mechanism & System & References \\
\hline RI & HJURP & $\begin{array}{l}\text { Cellular senescence } \\
\text { Cellular quiescence }\end{array}$ & $\begin{array}{l}\text { P53-dependent (?) } \\
\text { centromere identity }\end{array}$ & HDFs and HUVEC & Heo et al., 2013 \\
human RPE1 and starfish & Swartz et al., 2019 \\
oocyte & Mouse EBs, neural & Gao et al., 2018 \\
& & Listone eviction at promoters & differentiation & Gonzalez-Muñoz et al., 2014 \\
& ASF1A & Lineage differentiation & H9 ESCs, hADFs & Yang et al., 2011 \\
& ASF1A & Pluripotency/reprogramming & H3K56 acetylation & C2C12 to myotube & Song et al., 2012 \\
& ASF1A & Muscle differentiation & MyoD/MEF2 expression & C2C12 to osteoblast & Paul et al., 2016 \\
& ASF1A & Osteoblast conversion & H3.3 deposition & Human beta-cells & Messiaen et al., 2016
\end{tabular}

Summary of histone or histone chaperone roles in cell fate decisions in context of the systems studied and illuminated mechanisms.

\begin{tabular}{|c|c|}
\hline $\begin{array}{l}\text { FIGURE } 3 \text { | H3 enrichment depeno } \\
\text { become enriched in H3.3. After act } \\
\text { progenitors terminally differentiate in } \\
\text { maintained at the centromere durin } \\
\text { indicated in Figure 1. After termina }\end{array}$ & $\begin{array}{l}\text { otency } \\
\text { High } \mathrm{H} \text { 3.1 / 3.2 - H4 dimer } \\
\text { ds on the proliferative state and cellular potency. Adult stem cells remain in a quiescent state. Without replication these cells } \\
\text { tivation, stem cells differentiate into progenitors and rapidly expand, becoming more enriched in H3.1/2 histone. After expansion, } \mathrm{H} \text {, dimer } \\
\text { nto post-mitotic cells. Without replication, these cells also become enriched in H3.3. In quiescent cells, CENPA is actively } \\
\text { al differentiation, post-mitotic cell centromeres can lose stability over time. }\end{array}$ \\
\hline
\end{tabular}

example, CENPA in quiescent cells is specifically maintained to preserve proliferative potential (Figure 3; Swartz et al., 2019). Additionally, many cellular differentiation paradigms implicate CAF-1 and HIRA as a transcriptional repressor or activator, respectively (Figure $\mathbf{2 B}$ and Table $\mathbf{1}$ ).

Volk et al. demonstrated that while complete loss of CAF-1 in the mouse inhibits normal hematopoiesis, its reduced levels is tolerated (Volk et al., 2018). Low levels of CAF-1 protect the mice from cancer progression by triggering differentiation of MLL/AF9 leukemic cells into mature myeloid cells. In this setting, CAF-1 is proposed to maintain leukemic cell identity via its RD nucleosome assembly activity as well as its competitive binding to sites of myeloid specifying transcription factors.

In a screen for chromatin regulators, silencing the CD4 gene in CD8 + cytotoxic $\mathrm{T}$ cells, CAF-1 was also identified as a transcriptional repressor among other fork components, including MCM2 (Ng et al., 2019). In this setting, CAF-1, in addition to its nucleosome assembly function, is proposed to cooperate with DNA and histone modifying enzymes by binding directly to histone deacetylase and histone demethylases to ensure heritable silencing of the CD4 gene. More recently, single cell profiling demonstrates that CAF-1 loss in myeloid progenitor cells triggers their partial differentiation leading to a mixed cellular state (Preprint, Guo et al., 2020). Interestingly, in comparison to normal myeloid differentiation, CAF-1 loss triggers a unique chromatin accessibility environment and activation of multi-lineage specific transcription factors. How the transcriptionally repressive role of CAF-1 in these systems is linked to its H3 deposition, alternative deposition of histone variants and/or recruitment of chromatin regulators remain to be determined.

In contrast to CAF-1, HIRA is widely studied in various cellular models including several mesoderm-derived tissues as well as during neurogenesis (Figure 2B and Table 1). For example, during normal hematopoiesis, Chen et al. (2020) demonstrated that upon conditional deletion of HIRA in 
the mouse, long term hematopoietic stem cell (LT-HSC) function is impaired, leading to lethality. Interestingly, LTHSC is thought to be in a more quiescent state. Accordingly, HIRA deletion had no effect in fetal hematopoiesis where hematopoietic stem cells are actively cycling. As seen with the mature oocyte and the early zygote, it is tempting to speculate that H3.3 deposition by HIRA could be involved in maintaining a unique chromatin environment in LT-HSCs that is subsequently remodeled during their self-renewal and differentiation (see model in Figure 3). HIRA is also required to maintain leukemic cells. Majumder et al. (2019) show that down-regulation of HIRA in chronic myeloid leukemia leads to a differentiation phenotype and implanting HIRA KO progenitors results in increased megakaryocyte differentiation. In this context, depletion of HIRA causes enrichment of H3.3 at promoters of key megakaryocyte differentiation factors GATA2 and MKL1, and a loss of H3.3 at erythroid differentiation promoters. It will be intriguing to dissect the mechanisms of differential H3.3 deposition upon HIRA loss. In addition to hematopoiesis, HIRA and ASF1 have been implicated in C2C12 myoblast cellular plasticity. Both HIRA and ASF1 are important for myoblast differentiation into myotubes and for their osteogenic conversion (Yang et al., 2011; Song et al., 2012). In myoblast differentiation HIRA and ASF1 drive MyoD expression and allow for $\mathrm{H} 3.3$ accumulation at critical enhancer regions. Additional studies implicate the role of HIRA in neurogenesis showing that it can interact with B-catenin to promote neurogenesis ( $\mathrm{Li}$ and Jiao, 2017). In addition, in vivo conditional deletion of HIRA causes widespread defects in neurogenesis (Jeanne et al., 2021). Taken together in these systems, the HIRA mediated $\mathrm{H} 3$ deposition mechanisms are poorly characterized.

Interestingly, a recent study highlights HIRA's gap filling mechanisms in the context of metastatic transformation of breast and colon cancer tissues (Gomes et al., 2019) (Figure 1A). In this setting, the activation of epithelial-to-mesenchymal transition genes to promote metastasis is dependent on downregulation of canonical H3 deposition of CAF-1 and HIRA-mediated $\mathrm{H} 3.3$ deposition at regulatory sites. It will be interesting to determine the mechanism underlying the specific deposition of H3.3 at these sites. Given HIRA's selective deposition of $\mathrm{H} 3.3$ to regulatory elements and the body of active genes, HIRA's function as a histone chaperone in addition to H3.3S31 specific phosphorylation could create a chromatin environment to facilitate the binding of transcription factors and chromatin regulators to maintain cell identity or instruct cell fate change depending on the cellular context and environment. Interestingly, $\mathrm{H} 3.3$ is required for neuronal stem cell proliferation and differentiation via promoting $\mathrm{H} 3 \mathrm{~K} 16$ acetylation. Whether H3.3S31 is required in this context remains to be explored (Xia and Jiao, 2017).

Another example of $\mathrm{H} 3.3$ guided recruitment of chromatin factors was recently highlighted while dissecting the transcriptional response to pathogens. In this context, selective phosphorylation of $\mathrm{H} 3.3 \mathrm{~S} 31$ at rapidly induced genes triggers a chromatin signaling cascade via recruiting a histone methyltransferase that promotes transcriptional elongation and repulsing a chromatin reader that inhibits transcription
(Thorne et al., 2012; Guo et al., 2014; Wen H. et al., 2014; Armache et al., 2020).

Considering other $\mathrm{H} 3.3$ deposition pathways, conditional deletion of DAXX in pancreatic tissues supports its role in ERV silencing (Figure 2B and Table 1; Wasylishen et al., 2020). While no phenotypes were observed, the more permissible transcriptional state is proposed to increase responses to stressors and to impair recovery. Outside of DAXX function at repetitive elements, another study in neurons reported a non-canonical DAXX mediated H3.3 deposition at regulatory elements that is linked to neuronal activation (Michod et al., 2012). Furthermore, DAXX is responsible for the ectopic deposition of overexpressed CENPA which is a hallmark of many cancers (Figure 1D; Sharma et al., 2019). It will be interesting to probe how DAXX responds to the loss of HIRA in these systems and investigate the function of other histone chaperone mediated deposition of $\mathrm{H} 3$, including ASF1A/B, HJURP, SPT6 in these cellular settings.

\section{DISCUSSION}

Histone chaperones are in place to modulate the deposition of histones at the right place and right time and coordinate the action of accompanying chromatin factors, including lineage-specific transcription factors during quiescence, stem cell self-renewal, differentiation, or reprogramming. While the expression of histone variants during the cell cycle and development is well documented, the activity, complex diversity, and interplay of histone chaperones during these processes is poorly understood, especially in the context of cell fate transitions. This is clearly complicated by the multifunctional characteristics of histone chaperones as they play additional roles independent of nucleosome assembly that are in turn linked to chromatin regulation.

The lessons that we learned from studying $\mathrm{H} 3$ deposition pathways in the context of normal development, culture model and disease state suggest that the RD and RI H3 deposition pathways act in a balanced manner to maintain lineage identity and instruct cell fate change in response to signals. Aside from the traditional culture models, it will be interesting to exploit newly developed organoid culture models and gametogenesis platforms to characterize the mechanisms of histone exchange and apply the lessons we learned from model organisms (Figure 2A). These emerging culture models provide unique systems to perform biochemical studies and create high resolution spatiotemporal maps of histone deposition in the context of cell fate determination. Finally, future therapeutic avenues include (1) the identification of unique histone deposition machinery in disease states and investigating the epigenetic addictions as a consequence of histone mutations or compromised histone chaperone activity and (2) manipulate histone chaperone pathways to generate specific cell types for regenerative purposes.

\section{AUTHOR CONTRIBUTIONS}

SC proposed the topic of the review and outlined the structure. SC and JM mentored RF throughout the analysis and discussions. 
SC, JM, and RF reviewed the literature, wrote the review, designed the figures, and compiled the table. All authors contributed to the article and approved the submitted version.

\section{FUNDING}

This work was supported by the UC Cancer Research Coordinating Committee (CRCC - C21CR2175) and the City of Hope - UC Riverside Biomedical Research Initiative seed

\section{REFERENCES}

Abascal, F., Corpet, A., Gurard-Levin, Z. A., Juan, D., Ochsenbein, F., Rico, D., et al. (2013). Subfunctionalization via Adaptive Evolution Influenced by Genomic Context: The Case of Histone Chaperones ASF1a and ASF1b. Mole. Biol. Evol. 30, 1853-1866. doi: 10.1093/molbev/mst086

Adli, M. (2018). The CRISPR tool kit for genome editing and beyond. Nat. Commun. 9:1911. doi: 10.1038/s41467-018-04252-2

Ahmad, K., and Henikoff, S. (2002). Histone H3 variants specify modes of chromatin assembly. PNAS 99, 16477-16484. doi: 10.1073/pnas.17240 3699

Akiyama, T., Suzuki, O., Matsuda, J., and Aoki, F. (2011). Dynamic Replacement of Histone H3 Variants Reprograms Epigenetic Marks in Early Mouse Embryos. PLoS Genetics 7:e1002279. doi: 10.1371/journal.pgen.100 2279

Almouzni, G., and Méchali, M. (1988). Assembly of spaced chromatin promoted by DNA synthesis in extracts from Xenopus eggs. EMBO J. 7, 665-672. doi: 10.1002/j.1460-2075.1988.tb02861.x

Anzalone, A. V., Koblan, L. W., and Liu, D. R. (2020). Genome editing with CRISPR-Cas nucleases, base editors, transposases and prime editors. Nat. Biotechnol. 38, 824-844. doi: 10.1038/s41587-020-0561-9

Arimura, Y., Shirayama, K., Horikoshi, N., Fujita, R., Taguchi, H., Kagawa, W., et al. (2014). Crystal structure and stable property of the cancer-associated heterotypic nucleosome containing CENP-A and H3.3. Sci. Rep. 4:7115. doi: 10.1038/srep07115

Armache, A., Yang, S., Martínez, de Paz, A., Robbins, L. E., Durmaz, C., et al. (2020). Histone H3.3 phosphorylation amplifies stimulation-induced transcription. Nature 583, 852-857. doi: 10.1038/s41586-020-2533-0

Banaszynski, L. A., Wen, D., Dewell, S., Whitcomb, S. J., Lin, M., Diaz, N., et al. (2013). Hira-Dependent Histone H3.3 Deposition Facilitates PRC2 Recruitment at Developmental Loci in ES Cells. Cell 155, 107-120. doi: 10.1016/ j.cell.2013.08.061

Bellelli, R., Belan, O., Pye, V. E., Clement, C., Maslen, S. L., Skehel, J. M., et al. (2018). POLE3-POLE4 Is a Histone H3-H4 Chaperone that Maintains Chromatin Integrity during DNA Replication. Mol. Cell 72, 112.e-126.e. doi: 10.1016/j.molcel.2018.08.043

Bobkov, G. O. M., Huang, A., van den Berg, S. J. W., Mitra, S., Anselm, E., Lazou, V., et al. (2020). Spt6 is a maintenance factor for centromeric CENP-A. Nat. Commun. 11:2919. doi: 10.1038/s41467-02016695-7

Brumbaugh, J., Stefano, B. D., and Hochedlinger, K. (2019). Reprogramming: identifying the mechanisms that safeguard cell identity. Development 146:dev.182170. doi: 10.1242/dev.182170

Buenrostro, J. D., Giresi, P. G., Zaba, L. C., Chang, H. Y., and Greenleaf, W. J. (2013). Transposition of native chromatin for fast and sensitive epigenomic profiling of open chromatin, DNA-binding proteins and nucleosome position. Nat. Methods 10, 1213-1218. doi: 10.1038/nmeth. 2688

Burton, A., Brochard, V., Galan, C., Ruiz-Morales, E. R., Rovira, Q., Rodriguez-Terrones, D., et al. (2020). Heterochromatin establishment during early mammalian development is regulated by pericentromeric RNA and characterized by non-repressive H3K9me3. Nat. Cell Biol. 22, 767-778. doi: 10.1038/s41556-020-0536-6 grants. RF is supported by UC Riverside's Eugene Cota-Robles Fellowship.

\section{ACKNOWLEDGMENTS}

The authors thank Dr. Qi Chen, Dr. Andrew Volk, and members of the SC and JM lab for critical reading of the manuscript. The authors apologize to colleagues whose work could not be cited owing to space limitations.

Buschbeck, M., and Hake, S. B. (2017). Variants of core histones and their roles in cell fate decisions, development and cancer. Nat. Rev. Mole. Cell Biol. 18, 299-314. doi: 10.1038/nrm.2016.166

Bush, K. M., Yuen, B. T., Barrilleaux, B. L., Riggs, J. W., O’Geen, H., Cotterman, R. F., et al. (2013). Endogenous mammalian histone H3.3 exhibits chromatinrelated functions during development. Epigenet. Chromatin 6:7. doi: 10.1186/ 1756-8935-6-7

Champroux, A., Cocquet, J., Henry-Berger, J., Drevet, J. R., and Kocer, A. (2018). A Decade of Exploring the Mammalian Sperm Epigenome: Paternal Epigenetic and Transgenerational Inheritance. Front. Cell Dev. Biol. 6:00050. doi: 10.3389/ fcell.2018.00050

Cheloufi, S., Elling, U., Hopfgartner, B., Jung, Y. L., Murn, J., Ninova, M., et al. (2015). The histone chaperone CAF-1 safeguards somatic cell identity. Nature 528, 218-224. doi: 10.1038/nature15749

Cheloufi, S., and Hochedlinger, K. (2017). Emerging roles of the histone chaperone CAF-1 in cellular plasticity. Curr. Opin. Genet. Dev. 46, 83-94. doi: 10.1016/j. gde.2017.06.004

Chen, C., Sun, M., Warzecha, C., Bachu, M., Dey, A., Wu, T., et al. (2020). HIRA, a DiGeorge Syndrome Candidate Gene, Confers Proper Chromatin Accessibility on HSCs and Supports All Stages of Hematopoiesis. Cell Reports 30, 2136.e-2149.e. doi: 10.1016/j.celrep.2020.01.062

Cheng, L., Zhang, X., Wang, Y., Gan, H., Xu, X., Lv, X., et al. (2019). Chromatin Assembly Factor 1 (CAF-1) facilitates the establishment of facultative heterochromatin during pluripotency exit. Nucleic Acids Res. 47, 11114-11131. doi: 10.1093/nar/gkz858

Cho, C., Jung-Ha, H., Willis, W. D., Goulding, E. H., Stein, P., Xu, Z., et al. (2003). Protamine 2 Deficiency Leads to Sperm DNA Damage and Embryo Death in Mice1. Biol. Reproduct. 69, 211-217. doi: 10.1095/biolreprod.102.015115

Cho, C., Willis, W. D., Goulding, E. H., Jung-Ha, H., Choi, Y.-C., Hecht, N. B., et al. (2001). Haploinsufficiency of protamine-1 or -2 causes infertility in mice. Nat. Genet. 28, 82-86. doi: 10.1038/ng0501-82

Clément, C., Orsi, G. A., Gatto, A., Boyarchuk, E., Forest, A., Hajj, B., et al. (2018). High-resolution visualization of $\mathrm{H} 3$ variants during replication reveals their controlled recycling. Nat. Commun. 9:3181. doi: 10.1038/s41467-018-05697-1

Corces, M. R., Trevino, A. E., Hamilton, E. G., Greenside, P. G., SinnottArmstrong, N. A., Vesuna, S., et al. (2017). An improved ATAC-seq protocol reduces background and enables interrogation of frozen tissues. Nat. Methods 14, 959-962. doi: 10.1038/nmeth.4396

Couldrey, C., Carlton, M. B. L., Nolan, P. M., Colledge, W. H., and Evans, M. J. (1999). A retroviral Gene Trap Insertion into the Histone 3.3A Gene Causes Partial Neonatal Lethality, Stunted Growth, Neuromuscular Deficits and Male Sub-fertility in Transgenic Mice. Hum. Mole. Genetics 8, 2489-2495. doi: 10. 1093/hmg/8.13.2489

Das, A., Smoak, E. M., Linares-Saldana, R., Lampson, M. A., and Black, B. E. (2017). Centromere inheritance through the germline. Chromosoma 126, 595-604. doi: 10.1007/s00412-017-0640-y

De Clerck, L., Taelman, J., Popovic, M., Willems, S., Van der Jeught, M., Heindryckx, B., et al. (2019). Untargeted histone profiling during naive conversion uncovers conserved modification markers between mouse and human. Sci. Rep. 9:17240. doi: 10.1038/s41598-019-53681-6

Dilg, D., Saleh, R. N. M., Phelps, S. E. L., Rose, Y., Dupays, L., Murphy, C., et al. (2016). HIRA is required for heart development and directly Regulates Tnni2 and Tnnt3. PLoS One 11:e0161096. doi: 10.1371/journal.pone.0161096 
Dilworth, S. M., Black, S. J., and Laskey, R. A. (1987). Two complexes that contain histones are required for nucleosome assembly in vitro: Role of nucleoplasmin and N1 in Xenopus egg extracts. Cell 51, 1009-1018. doi: 10.1016/00928674(87)90587-3

Dixon, J. R., Selvaraj, S., Yue, F., Kim, A., Li, Y., Shen, Y., et al. (2012). Topological domains in mammalian genomes identified by analysis of chromatin interactions. Nature 485, 376-380. doi: 10.1038/nature11082

Dunleavy, E. M., Almouzni, G., and Karpen, G. H. (2011). H3.3 is deposited at centromeres in S phase as a placeholder for newly assembled CENP-A in G1 phase. Nucleus 2, 146-157. doi: 10.4161/nucl.2.2.15211

Dunleavy, E. M., Roche, D., Tagami, H., Lacoste, N., Ray-Gallet, D., Nakamura, Y., et al. (2009). HJURP Is a Cell-Cycle-Dependent Maintenance and Deposition Factor of CENP-A at Centromeres. Cell 137, 485-497. doi: 10.1016/j.cell.2009. 02.040

Eckersley-Maslin, M. A., Alda-Catalinas, C., and Reik, W. (2018). Dynamics of the epigenetic landscape during the maternal-to-zygotic transition. Nat. Rev. Mole. Cell Biol. 19, 436-450. doi: 10.1038/s41580-018-0008-z

Elsässer, S. J., Noh, K.-M., Diaz, N., Allis, C. D., and Banaszynski, L. A. (2015). Histone $\mathrm{H} 3.3$ is required for endogenous retroviral element silencing in embryonic stem cells. Nature 522, 240-244. doi: 10.1038/nature14345

Erkek, S., Hisano, M., Liang, C.-Y., Gill, M., Murr, R., Dieker, J., et al. (2013). Molecular determinants of nucleosome retention at $\mathrm{CpG}$-rich sequences in mouse spermatozoa. Nat. Struct. Mole. Biol. 20, 868-875. doi: 10.1038/nsmb. 2599

Evano, B., Khalilian, S., Le Carrou, G., Almouzni, G., and Tajbakhsh, S. (2020). Dynamics of Asymmetric and Symmetric Divisions of Muscle Stem Cells In Vivo and on Artificial Niches. Cell Rep. 30, 3195.e-3206.e. doi: 10.1016/j.celrep. 2020.01.097

Evans, M. J., and Kaufman, M. H. (1981). Establishment in culture of pluripotential cells from mouse embryos. Nature 292, 154-156. doi: 10.1038/292154a0

Fang, H.-T., El Farran, C. A., Xing, Q. R., Zhang, L.-F., Li, H., Lim, B., et al. (2018). Global H3.3 dynamic deposition defines its bimodal role in cell fate transition. Nat. Commun. 9:1537. doi: 10.1038/s41467-018-03904-7

Filipescu, D., Müller, S., and Almouzni, G. (2014). Histone H3 Variants and Their Chaperones During Development and Disease: Contributing to Epigenetic Control. Ann. Rev. Cell Dev. Biol. 30, 615-646. doi: 10.1146/annurev-cellbio100913-013311

Franklin, S. G., and Zweidler, A. (1977). Non-allelic variants of histones 2a, 2b and 3 in mammals. Nature 266, 273-275. doi: 10.1038/266273a0

Gao, Y., Gan, H., Lou, Z., and Zhang, Z. (2018). Asfla resolves bivalent chromatin domains for the induction of lineage-specific genes during mouse embryonic stem cell differentiation. PNAS 115, E6162-E6171. doi: 10.1073/ pnas. 1801909115

Gehre, M., Bunina, D., Sidoli, S., Lübke, M. J., Diaz, N., Trovato, M., et al. (2020). Lysine 4 of histone H3.3 is required for embryonic stem cell differentiation, histone enrichment at regulatory regions and transcription accuracy. Nat. Genet. 52, 273-282. doi: 10.1038/s41588-020-0586-5

Goldberg, A. D., Banaszynski, L. A., Noh, K.-M., Lewis, P. W., Elsaesser, S. J., Stadler, S., et al. (2010). Distinct Factors Control Histone Variant H3.3 Localization at Specific Genomic Regions. Cell 140, 678-691. doi: 10.1016/j.cell. 2010.01.003

Gomes, A. P., Ilter, D., Low, V., Rosenzweig, A., Shen, Z.-J., Schild, T., et al. (2019). Dynamic Incorporation of Histone H3 Variants into Chromatin Is Essential for Acquisition of Aggressive Traits and Metastatic Colonization. Cancer Cell 36, 402.e-417.e. doi: 10.1016/j.ccell.2019. 08.006

Gonzalez-Muñoz, E., Arboleda-Estudillo, Y., Out, H. H., and Cibelli, J. B. (2014). Histone chaperone ASF1A is required for maintenance of pluripotency and cellular reprogramming. Science 2014:1254745. doi: 10.1126/science.1254745

Gou, L.-T., Lim, D.-H., Ma, W., Aubol, B. E., Hao, Y., Wang, X., et al. (2020). Initiation of Parental Genome Reprogramming in Fertilized Oocyte by Splicing Kinase SRPK1-Catalyzed Protamine Phosphorylation. Cell 180, 1212.e-1227.e. doi: $10.1016 /$ j.cell.2020.02.020

Groth, A., Corpet, A., Cook, A. J. L., Roche, D., Bartek, J., Lukas, J., et al. (2007). Regulation of Replication Fork Progression Through Histone Supply and Demand. Science 318, 1928-1931. doi: 10.1126/science.114 8992
Grover, P., Asa, J. S., and Campos, E. I. (2018). H3-H4 Histone Chaperone Pathways. Ann. Rev. Genet. 52, 109-130. doi: 10.1146/annurev-genet-120417031547

Guo, R., Zheng, L., Park, J. W., Lv, R., Chen, H., Jiao, F., et al. (2014). BS69/ZMYND11 Reads and Connects Histone H3.3 Lysine 36 TrimethylationDecorated Chromatin to Regulated Pre-mRNA Processing. Mole. Cell 56, 298-310. doi: 10.1016/j.molcel.2014.08.022

Guo, Y., Ji, F., Murn, J., Frankhouser, D., Blanco, M. A., Chiem, C., et al. (2020). The Histone Chaperone CAF-1 Sustains Myeloid Lineage Identity. bioRxiv 2020:350447. doi: 10.1101/2020.10.22.350447

Hamazaki, N., Kyogoku, H., Araki, H., Miura, F., Horikawa, C., Hamada, N., et al. (2021). Reconstitution of the oocyte transcriptional network with transcription factors. Nature 589, 264-269. doi: 10.1038/s41586-020-3027-9

Hammond, C. M., Strømme, C. B., Huang, H., Patel, D. J., and Groth, A. (2017). Histone chaperone networks shaping chromatin function. Nat. Rev. Mole. Cell Biol. 18, 141-158. doi: 10.1038/nrm.2016.159

Hammoud, S. S., Nix, D. A., Zhang, H., Purwar, J., Carrell, D. T., and Cairns, B. R. (2009). Distinctive chromatin in human sperm packages genes for embryo development. Nature 460, 473-478. doi: 10.1038/nature08162

Hartford, S. A., Luo, Y., Southard, T. L., Min, I. M., Lis, J. T., and Schimenti, J. C. (2011). Minichromosome maintenance helicase paralog MCM9 is dispensible for DNA replication but functions in germ-line stem cells and tumor suppression. PNAS 108, 17702-17707. doi: 10.1073/pnas.1113524108

He, Q., Kim, H., Huang, R., Lu, W., Tang, M., Shi, F., et al. (2015). The Daxx/Atrx Complex Protects Tandem Repetitive Elements during DNA Hypomethylation by Promoting H3K9 Trimethylation. Cell Stem Cell 17, 273-286. doi: 10.1016/j. stem.2015.07.022

Heo, J.-I., Cho, J. H., and Kim, J.-R. (2013). HJURP regulates cellular senescence in human fibroblasts and endothelial cells via a p53-dependent pathway. $J$. Gerontol. A Biol. Sci. Med. Sci. 68, 914-925. doi: 10.1093/gerona/gls257

Hewish, D. R., and Burgoyne, L. A. (1973). Chromatin sub-structure. The digestion of chromatin DNA at regularly spaced sites by a nuclear deoxyribonuclease. Biochem. Biophys. Res. Commun. 52, 504-510. doi: 10.1016/0006-291X(73) 90740-7

Hoelper, D., Huang, H., Jain, A. Y., Patel, D. J., and Lewis, P. W. (2017). Structural and mechanistic insights into ATRX-dependent and -independent functions of the histone chaperone DAXX. Nat. Commun. 8:1193. doi: 10.1038/s41467-01701206-y

Houlard, M., Berlivet, S., Probst, A. V., Quivy, J.-P., Héry, P., Almouzni, G., et al. (2006). CAF-1 Is Essential for Heterochromatin Organization in Pluripotent Embryonic Cells. PLoS Genetics 2:e181. doi: 10.1371/journal.pgen.0020181

Howman, E. V., Fowler, K. J., Newson, A. J., Redward, S., MacDonald, A. C., Kalitsis, P., et al. (2000). Early disruption of centromeric chromatin organization in centromere protein A (Cenpa) null mice. PNAS 97, 1148-1153. doi: 10.1073/pnas.97.3.1148

Huang, H., Strømme, C. B., Saredi, G., Hödl, M., Strandsby, A., González-Aguilera, C., et al. (2015). A unique binding mode enables MCM2 to chaperone histones H3-H4 at replication forks. Nat. Struct. Mole. Biol. 22, 618-626. doi: 10.1038/ nsmb. 3055

Ishiuchi, T., Abe, S., Inoue, K., Yeung, W. K. A., Miki, Y., Ogura, A., et al. (2021). Reprogramming of the histone H3.3 landscape in the early mouse embryo. Nat. Struct. Mole. Biol. 28, 38-49. doi: 10.1038/s41594-020-00521-1

Ishiuchi, T., Enriquez-Gasca, R., Mizutani, E., Bošković, A., Ziegler-Birling, C., Rodriguez-Terrones, D., et al. (2015). Early embryonic-like cells are induced by downregulating replication-dependent chromatin assembly. Nat. Struct. Mol. Biol. 22, 662-671. doi: 10.1038/nsmb.3066

Jang, C.-W., Shibata, Y., Starmer, J., Yee, D., and Magnuson, T. (2015). Histone H3.3 maintains genome integrity during mammalian development. Genes Dev. 29, 1377-1392. doi: 10.1101/gad.264150.115

Jansen, L. E. T., Black, B. E., Foltz, D. R., and Cleveland, D. W. (2007). Propagation of centromeric chromatin requires exit from mitosis. J. Cell Biol. 176, 795-805. doi: $10.1083 /$ jcb.200701066

Jeanne, M., Vuillaume, M.-L., Ung, D. C., Vancollie, V. E., Wagner, C., Collins, S. C., et al. (2021). Haploinsufficiency of the HIRA gene located in the 22q11 deletion syndrome region is associated with abnormal neurodevelopment and impaired dendritic outgrowth. Hum. Genet. 2021, 2252-2251. doi: 10.1007/ s00439-020-02252-1 
Jeronimo, C., Poitras, C., and Robert, F. (2019). Histone Recycling by FACT and Spt6 during Transcription Prevents the Scrambling of Histone Modifications. Cell Rep. 28, 1206.e-1218.e. doi: 10.1016/j.celrep.2019.06.097

Kaplan, C. D., Laprade, L., and Winston, F. (2003). Transcription Elongation Factors Repress Transcription Initiation from Cryptic Sites. Science 301, 10961099. doi: $10.1126 /$ science. 1087374

Kaya-Okur, H. S., Wu, S. J., Codomo, C. A., Pledger, E. S., Bryson, T. D., Henikoff, J. G., et al. (2019). CUT\&Tag for efficient epigenomic profiling of small samples and single cells. Nat. Commun. 10:1930. doi: 10.1038/s41467-019-09 982-5

Kong, S., Han, X., Cui, T., Zhou, C., Jiang, Y., Zhang, H., et al. (2016). MCM2 mediates progesterone-induced endometrial stromal cell proliferation and differentiation in mice. Endocrine 53, 595-606. doi: 10.1007/s12020-0160894-9

Kornberg, R. D. (1974). Chromatin Structure: A Repeating Unit of Histones and DNA. Science 184, 868-871. doi: 10.1126/science.184.4139.868

Lacoste, N., Woolfe, A., Tachiwana, H., Garea, A. V., Barth, T., Cantaloube, S., et al. (2014). Mislocalization of the centromeric histone variant CenH3/CENP-A in human cells depends on the chaperone DAXX. Mol. Cell 53, 631-644. doi: 10.1016/j.molcel.2014.01.018

Laskey, R. A., Honda, B. M., Mills, A. D., and Finch, J. T. (1978). Nucleosomes are assembled by an acidic protein which binds histones and transfers them to DNA. Nature 275, 416-420. doi: 10.1038/275416a0

Laskey, R. A., Mills, A. D., and Morris, N. R. (1977). Assembly of SV40 chromatin in a cell-free system from Xenopus eggs. Cell 10, 237-243. doi: 10.1016/00928674(77)90217-3

Lewis, P. W., Elsaesser, S. J., Noh, K.-M., Stadler, S. C., and Allis, C. D. (2010). Daxx is an H3.3-specific histone chaperone and cooperates with ATRX in replicationindependent chromatin assembly at telomeres. PNAS 107, 14075-14080. doi: 10.1073 /pnas. 1008850107

Li, Y., and Jiao, J. (2017). Histone chaperone HIRA regulates neural progenitor cell proliferation and neurogenesis via $\beta$-catenin. J. Cell Biol. 216, 1975-1992. doi: $10.1083 /$ jcb.201610014

Lin, C.-J., Koh, F. M., Wong, P., Conti, M., and Ramalho-Santos, M. (2014). Hiramediated H3.3 incorporation is required for DNA replication and ribosomal RNA transcription in the mouse zygote. Dev. Cell 30, 268-279. doi: 10.1016/j. devcel.2014.06.022

Loppin, B., and Berger, F. (2020). Histone Variants: The Nexus of Developmental Decisions and Epigenetic Memory. Annu. Rev. Genet. 54, 121-149. doi: 10.1146/ annurev-genet-022620-100039

Loppin, B., Bonnefoy, E., Anselme, C., Laurençon, A., Karr, T. L., and Couble, P. (2005). The histone H3.3 chaperone HIRA is essential for chromatin assembly in the male pronucleus. Nature 437, 1386-1390. doi: 10.1038/nature04059

Lorain, S., Quivy, J.-P., Monier-Gavelle, F., Scamps, C., Lécluse, Y., Almouzni, G., et al. (1998). Core Histones and HIRIP3, a Novel Histone-Binding Protein, Directly Interact with WD Repeat Protein HIRA. Mole. Cell. Biol. 18, 55465556. doi: 10.1128/MCB.18.9.5546

Luger, K., Mäder, A. W., Richmond, R. K., Sargent, D. F., and Richmond, T. J. (1997). Crystal structure of the nucleosome core particle at $2.8 \AA$ resolution. Nature 389, 251-260. doi: 10.1038/38444

Luzzati, V., and Nicolaieff, A. (1959). Etude par diffusion des rayons X aux petits angles des gels d'acide désoxyribonucléique et de nucléoprotéines: (note préliminaire). J. Mole. Biol. 1, 127-IN5. doi: 10.1016/S0022-2836(59)80041-3

Ma, B., Trieu, T.-J., Cheng, J., Zhou, S., Tang, Q., Xie, J., et al. (2020). Differential Histone Distribution Patterns in Induced Asymmetrically Dividing Mouse Embryonic Stem Cells. Cell Rep. 32:108003. doi: 10.1016/j.celrep.2020.108003

Macfarlan, T. S., Gifford, W. D., Driscoll, S., Lettieri, K., Rowe, H. M., Bonanomi, D., et al. (2012). Embryonic stem cell potency fluctuates with endogenous retrovirus activity. Nature 487, 57-63. doi: 10.1038/nature11244

Magnaghi, P., Roberts, C., Lorain, S., Lipinski, M., and Scambler, P. J. (1998). HIRA, a mammalian homologue of Saccharomyces cerevisiae transcriptional co-repressors, interacts with Pax3. Nat. Genet. 20, 74-77. doi: 10.1038/1739

Majumder, A., Dharan, A. T., Baral, I., Varghese, P. C., Mukherjee, A., Subhadradevi, L., et al. (2019). Histone chaperone HIRA dictate proliferation vs differentiation of chronic myeloid leukemia cells. FASEB BioAdv. 1, 525-537. doi: 10.1096/fba.2019-00014

Martin, G. R. (1981). Isolation of a pluripotent cell line from early mouse embryos cultured in medium conditioned by teratocarcinoma stem cells. Proc. Natl. Acad. Sci. 78, 7634-7638. doi: 10.1073/pnas.78.12. 7634

Martire, S., and Banaszynski, L. A. (2020). The roles of histone variants in finetuning chromatin organization and function. Nat. Rev. Mole. Cell Biol. 21, 522-541. doi: 10.1038/s41580-020-0262-8

Martire, S., Gogate, A. A., Whitmill, A., Tafessu, A., Nguyen, J., Teng, Y.-C., et al. (2019). Phosphorylation of histone H3.3 at serine 31 promotes $\mathrm{p} 300$ activity and enhancer acetylation. Nat. Genet. 51, 941-946. doi: 10.1038/s41588-019-0428-5

Meers, M. P., Bryson, T. D., Henikoff, J. G., and Henikoff, S. (2019). Improved CUT\&RUN chromatin profiling tools. eLife 8:e46314. doi: 10.7554/eLife.46314

Mendiratta, S., Gatto, A., and Almouzni, G. (2018). Histone supply: Multitiered regulation ensures chromatin dynamics throughout the cell cycle. J. Cell Biol. 218, 39-54. doi: 10.1083/jcb.201807179

Messiaen, S., Guiard, J., Aigueperse, C., Fliniaux, I., Tourpin, S., Barroca, V., et al. (2016). Loss of the histone chaperone ASF1B reduces female reproductive capacity in mice. Reproduction 151, 477-489. doi: 10.1530/REP-15-0327

Michaelson, J. S., Bader, D., Kuo, F., Kozak, C., and Leder, P. (1999). Loss of Daxx, a promiscuously interacting protein, results in extensive apoptosis in early mouse development. Genes Dev. 13, 1918-1923. doi: 10.1101/gad.13.15.1918

Michod, D., Bartesaghi, S., Khelifi, A., Bellodi, C., Berliocchi, L., Nicotera, P., et al. (2012). Calcium-Dependent Dephosphorylation of the Histone Chaperone DAXX Regulates H3.3 Loading and Transcription upon Neuronal Activation. Neuron 74, 122-135. doi: 10.1016/j.neuron.2012.02.021

Muhire, B. M., Booker, M. A., and Tolstorukov, M. Y. (2019). Non-neutral evolution of H3.3-encoding genes occurs without alterations in protein sequence. Sci. Rep. 9:8472. doi: 10.1038/s41598-019-44800-4

Nakano, S., Stillman, B., and Horvitz, H. R. (2011). CAF-1-Mediated Chromatin Assembly Generates a Bilateral Asymmetry in C. elegans Neuroanatomy. Cell 147, 1525-1536. doi: 10.1016/j.cell.2011.11.053

Ng, C., Aichinger, M., Nguyen, T., Au, C., Najar, T., Wu, L., et al. (2019). The histone chaperone CAF-1 cooperates with the DNA methyltransferases to maintain Cd4 silencing in cytotoxic T cells. Genes Dev. 33, 669-683. doi: 10. 1101/gad.322024.118

Olins, A. L., and Olins, D. E. (1974). Spheroid Chromatin Units ( $v$ Bodies). Science 183, 330-332. doi: 10.1126/science.183.4122.330

Oudet, P., Gross-Bellard, M., and Chambon, P. (1975). Electron microscopic and biochemical evidence that chromatin structure is a repeating unit. Cell 4 , 281-300. doi: 10.1016/0092-8674(75)90149-X

Paul, P. K., Rabaglia, M. E., Wang, C.-Y., Stapleton, D. S., Leng, N., Kendziorski, C., et al. (2016). Histone chaperone ASF1B promotes human $\beta$-cell proliferation via recruitment of histone H3.3. Cell Cycle 15, 3191-3202. doi: 10.1080/15384101. 2016.1241914

Pérez-Palacios, R., and Bourc'his, D. (2018). A single-cell chromatin map of human embryos. Nat. Cell Biol. 20, 742-744. doi: 10.1038/s41556-018-0134-z

Petryk, N., Dalby, M., Wenger, A., Stromme, C. B., Strandsby, A., Andersson, R., et al. (2018). MCM2 promotes symmetric inheritance of modified histones during DNA replication. Science 361, 1389-1392. doi: 10.1126/science.aau0294

Probst, A. V., and Almouzni, G. (2011). Heterochromatin establishment in the context of genome-wide epigenetic reprogramming. Trends Genet. 27, 177-185. doi: 10.1016/j.tig.2011.02.002

Pruitt, S. C., Bailey, K. J., and Freeland, A. (2007). Reduced Mcm2 Expression Results in Severe Stem/Progenitor Cell Deficiency and Cancer. Stem Cells 25, 3121-3132. doi: 10.1634/stemcells.2007-0483

Raja, S. J., and Renkawitz-Pohl, R. (2005). Replacement by Drosophila melanogaster Protamines and Mst77F of Histones during Chromatin Condensation in Late Spermatids and Role of Sesame in the Removal of These Proteins from the Male Pronucleus. Mole. Cell. Biol. 25, 6165-6177. doi: 10. 1128/MCB.25.14.6165-6177.2005

Ray-Gallet, D., Quivy, J.-P., Scamps, C., Martini, E. M.-D., Lipinski, M., and Almouzni, G. (2002). HIRA Is Critical for a Nucleosome Assembly Pathway Independent of DNA Synthesis. Mole. Cell 9, 1091-1100. doi: 10.1016/S10972765(02)00526-9

Ray-Gallet, D., Woolfe, A., Vassias, I., Pellentz, C., Lacoste, N., Puri, A., et al. (2011). Dynamics of Histone H3 Deposition In Vivo Reveal a Nucleosome Gap-Filling Mechanism for H3.3 to Maintain Chromatin Integrity. Mole. Cell 44, 928-941. doi: 10.1016/j.molcel.2011.12.006

Roberts, C., Sutherland, H. F., Farmer, H., Kimber, W., Halford, S., Carey, A., et al. (2002). Targeted Mutagenesis of the Hira Gene Results in Gastrulation Defects 
and Patterning Abnormalities of Mesoendodermal Derivatives Prior to Early Embryonic Lethality. Mole. Cell. Biol. 22, 2318-2328. doi: 10.1128/MCB.22.7. 2318-2328.2002

Saleh, R. N. M., Dilg, D., Abou Zeid, A. A., Hashad, D. I., Scambler, P. J., and Chapgier, A. L. A. (2018). HIRA directly targets the enhancers of selected cardiac transcription factors during in vitro differentiation of mouse embryonic stem cells. Mol. Biol. Rep. 45, 1001-1011. doi: 10.1007/s11033-018-4247-z

Schlesinger, S., Kaffe, B., Melcer, S., Aguilera, J. D., Sivaraman, D. M., Kaplan, T., et al. (2017). A hyperdynamic H3.3 nucleosome marks promoter regions in pluripotent embryonic stem cells. Nucleic Acids Res. 45, 12181-12194. doi: $10.1093 / \mathrm{nar} / \mathrm{gkx} 817$

Schneiderman, J. I., Orsi, G. A., Hughes, K. T., Loppin, B., and Ahmad, K. (2012). Nucleosome-depleted chromatin gaps recruit assembly factors for the H3.3 histone variant. PNAS 109, 19721-19726. doi: 10.1073/pnas.120662 9109

Serra-Cardona, A., and Zhang, Z. (2018). Replication-Coupled Nucleosome Assembly in the Passage of Epigenetic Information and Cell Identity. Trends Biochem. Sci. 43, 136-148. doi: 10.1016/j.tibs.2017.12.003

Sharma, A. B., Dimitrov, S., Hamiche, A., and Van Dyck, E. (2019). Centromeric and ectopic assembly of CENP-A chromatin in health and cancer: old marks and new tracks. Nucleic Acids Res. 47, 1051-1069. doi: 10.1093/nar/gky1298

Sitbon, D., Boyarchuk, E., Dingli, F., Loew, D., and Almouzni, G. (2020). Histone variant H3.3 residue S31 is essential for Xenopus gastrulation regardless of the deposition pathway. Nat. Commun. 11:1256. doi: 10.1038/s41467-020-15084-4

Smith, S., and Stillman, B. (1989). Purification and characterization of CAF-I, a human cell factor required for chromatin assembly during DNA replication in vitro. Cell 58, 15-25. doi: 10.1016/0092-8674(89) 90398-X

Song, T.-Y., Yang, J.-H., Park, J. Y., Song, Y., Han, J.-W., Youn, H.-D., et al. (2012). The role of histone chaperones in osteoblastic differentiation of C2C12 myoblasts. Biochem. Biophys. Res. Commun. 423, 726-732. doi: 10.1016/j.bbrc. 2012.06.026

Struhl, K., and Segal, E. (2013). Determinants of nucleosome positioning. Nat. Struct. Mole. Biol. 20, 267-273. doi: 10.1038/nsmb.2506

Swartz, S. Z., McKay, L. S., Su, K.-C., Bury, L., Padeganeh, A., Maddox, P. S., et al. (2019). Quiescent Cells Actively Replenish CENP-A Nucleosomes to Maintain Centromere Identity and Proliferative Potential. Dev. Cell 51, 35.e-48.e. doi: 10.1016/j.devcel.2019.07.016

Talbert, P. B., and Henikoff, S. (2010). Histone variants - ancient wrap artists of the epigenome. Nat. Rev. Mole. Cell Biol. 11, 264-275. doi: 10.1038/nrm 2861

Tang, J., Wu, S., Liu, H., Stratt, R., Barak, O. G., Shiekhattar, R., et al. (2004). A Novel Transcription Regulatory Complex Containing Death Domainassociated Protein and the ATR-X Syndrome Protein. J. Biol. Chem. 279, 20369-20377. doi: 10.1074/jbc.M401321200

Tang, M. C. W., Jacobs, S. A., Mattiske, D. M., Soh, Y. M., Graham, A. N., Tran, A., et al. (2015). Contribution of the Two Genes Encoding Histone Variant H3.3 to Viability and Fertility in Mice. PLoS Genet. 11:e1004964. doi: 10.1371/journal. pgen. 1004964

Tang, M. C. W., Jacobs, S. A., Wong, L. H., and Mann, J. R. (2013). Conditional allelic replacement applied to genes encoding the histone variant $\mathrm{H} 3.3$ in the mouse. Genesis 51, 142-146. doi: 10.1002/dvg. 22366

Tang, Y., Poustovoitov, M. V., Zhao, K., Garfinkel, M., Canutescu, A., Dunbrack, R., et al. (2006). Structure of a human ASF1a-HIRA complex and insights into specificity of histone chaperone complex assembly. Nat. Struct. Mole. Biol. 13, 921-929. doi: 10.1038/nsmb1147

Thorne, J. L., Ouboussad, L., and Lefevre, P. F. (2012). Heterochromatin protein 1 gamma and IкB kinase alpha interdependence during tumour necrosis factor gene transcription elongation in activated macrophages. Nucleic Acids Res. 40, 7676-7689. doi: 10.1093/nar/gks509

Torné, J., Ray-Gallet, D., Boyarchuk, E., Garnier, M., Le Baccon, P., Coulon, A., et al. (2020). Two HIRA-dependent pathways mediate H3.3 de novo deposition and recycling during transcription. Nat. Struct. Mole. Biol. 27, 1057-1068. doi: 10.1038/s41594-020-0492-7

Torres-Flores, U., and Hernández-Hernández, A. (2020). The Interplay Between Replacement and Retention of Histones in the Sperm Genome. Front. Genet. 11:00780. doi: 10.3389/fgene.2020.00780

Torres-Padilla, M.-E., Bannister, A. J., Hurd, P. J., Kouzarides, T., and ZernickaGoetz, M. (2006). Dynamic distribution of the replacement histone variant H3.3 in the mouse oocyte and preimplantation embryos. Int. J. Dev. Biol. 50, 455-461. doi: $10.1387 / \mathrm{ijdb} .052073 \mathrm{mt}$

Tyler, J. K., Adams, C. R., Chen, S.-R., Kobayashi, R., Kamakaka, R. T., and Kadonaga, J. T. (1999). The RCAF complex mediates chromatin assembly during DNA replication and repair. Nature 402, 555-560. doi: 10.1038/990147

Valenzuela, N., Soibam, B., Li, L., Wang, J., Byers, L. A., Liu, Y., et al. (2017). HIRA deficiency in muscle fibers causes hypertrophy and susceptibility to oxidative stress. J. Cell Sci. 130, 2551-2563. doi: 10.1242/jcs. 200642

Venkatesh, S., and Workman, J. L. (2015). Histone exchange, chromatin structure and the regulation of transcription. Nat. Rev. Mole. Cell Biol. 16, 178-189. doi: 10.1038/nrm3941

Volk, A., Liang, K., Suraneni, P., Li, X., Zhao, J., Bulic, M., et al. (2018). A CHAF1BDependent Molecular Switch in Hematopoiesis and Leukemia Pathogenesis. Cancer Cell 34, 707.e-723.e. doi: 10.1016/j.ccell.2018.10.004

Vos, S. M., Farnung, L., Boehning, M., Wigge, C., Linden, A., Urlaub, H., et al. (2018). Structure of activated transcription complex Pol II-DSIF-PAF-SPT6. Nature 560, 607-612. doi: 10.1038/s41586-018-0440-4

Vos, S. M., Farnung, L., Linden, A., Urlaub, H., and Cramer, P. (2020). Structure of complete Pol II-DSIF-PAF-SPT6 transcription complex reveals RTF1 allosteric activation. Nat. Struct. Mole. Biol. 27, 668-677. doi: 10.1038/s41594-0200437-1

Wasylishen, A. R., Sun, C., Moyer, S. M., Qi, Y., Chau, G. P., Aryal, N. K., et al. (2020). Daxx maintains endogenous retroviral silencing and restricts cellular plasticity in vivo. Sci. Adv. 6:eaba8415. doi: 10.1126/sciadv.aba8415

Wen, D., Banaszynski, L. A., Liu, Y., Geng, F., Noh, K.-M., Xiang, J., et al. (2014). Histone variant $\mathrm{H} 3.3$ is an essential maternal factor for oocyte reprogramming. Proc. Natl. Acad. Sci. 111, 7325-7330. doi: 10.1073/pnas.1406389111

Wen, H., Li, Y., Xi, Y., Jiang, S., Stratton, S., Peng, D., et al. (2014). ZMYND11 links histone $\mathrm{H} 3.3 \mathrm{~K} 36 \mathrm{me} 3$ to transcription elongation and tumour suppression. Nature 508, 263-268. doi: 10.1038/nature13045

Wu, C., Ding, H., Wang, S., Li, Y., Liu, S.-B., Wang, X., et al. (2020). DAXX inhibits cancer stemness and epithelial-mesenchymal transition in gastric cancer. Br. J. Cancer 122, 1477-1485. doi: 10.1038/s41416-020-0800-3

Wilkins, M. H. F., Zubay, G., and Wilson, H. R. (1959). X-ray diffraction studies of the molecular structure of nucleohistone and chromosomes. J. Mole. Biol. 1, 179-IN10. doi: 10.1016/S0022-2836(59)80046-2

Xia, W., and Jiao, J. (2017). Histone variant H3.3 orchestrates neural stem cell differentiation in the developing brain. Cell Death Diff. 24, 1548-1563. doi: $10.1038 /$ cdd. 2017.77

Yang, J.-H., Song, Y., Seol, J.-H., Park, J. Y., Yang, Y.-J., Han, J.-W., et al. (2011). Myogenic transcriptional activation of MyoD mediated by replication-independent histone deposition. PNAS 108, 85-90. doi: 10.1073/ pnas. 1009830108

Yang, J.-H., Song, T.-Y., Jo, C., Park, J., Lee, H.-Y., Song, I., et al. (2016). Differential regulation of the histone chaperone HIRA during muscle cell differentiation by a phosphorylation switch. Exp. Mol. Med. 48, e252-e252. doi: 10.1038/emm. 2016.68

Yoshida, K., Muratani, M., Araki, H., Miura, F., Suzuki, T., Dohmae, N., et al. (2018). Mapping of histone-binding sites in histone replacement-completed spermatozoa. Nat. Commun. 9:3885. doi: 10.1038/s41467-018-06243-9

Yuen, B. T. K., Bush, K. M., Barrilleaux, B. L., Cotterman, R., and Knoepfler, P. S. (2014). Histone H3.3 regulates dynamic chromatin states during spermatogenesis. Development 141, 3483-3494. doi: 10.1242/dev.106450

Zasadziñska, E., Huang, J., Bailey, A. O., Guo, L. Y., Lee, N. S., Srivastava, S., et al. (2018). Inheritance of CENP-A Nucleosomes during DNA Replication Requires HJURP. Dev. Cell 47, 348.e-362.e. doi: 10.1016/j.devcel.2018.09.003

Zhu, Z., Li, C., Zeng, Y., Ding, J., Qu, Z., Gu, J., et al. (2017). PHB Associates with the HIRA Complex to Control an Epigenetic-Metabolic Circuit in Human ESCs. Cell Stem Cell 20, 274.e-289.e. doi: 10.1016/j.stem.2016.11.002

Conflict of Interest: The authors declare that the research was conducted in the absence of any commercial or financial relationships that could be construed as a potential conflict of interest.

Copyright (c) 2021 Franklin, Murn and Cheloufi. This is an open-access article distributed under the terms of the Creative Commons Attribution License (CC BY). The use, distribution or reproduction in other forums is permitted, provided the original author(s) and the copyright owner(s) are credited and that the original publication in this journal is cited, in accordance with accepted academic practice. No use, distribution or reproduction is permitted which does not comply with these terms. 